\title{
Stable exponential cosmological solutions with three different Hubble-like parameters in EGB model with a $\Lambda$-term
}

\author{
K. K. Ernazarov ${ }^{1}$, V. D. Ivashchuk ${ }^{1,2, a}$ \\ ${ }^{1}$ Institute of Gravitation and Cosmology, Peoples' Friendship University of Russia (RUDN University), 6 Miklukho-Maklaya Street, Moscow \\ 117198, Russian Federation \\ ${ }^{2}$ Center for Gravitation and Fundamental Metrology, VNIIMS, 46 Ozyornaya Street, Moscow 119361, Russian Federation
}

Received: 30 March 2020 / Accepted: 2 June 2020 / Published online: 16 June 2020

(C) The Author(s) 2020

\begin{abstract}
We consider a $D$-dimensional Einstein-GaussBonnet model with a cosmological term $\Lambda$ and two non-zero constants: $\alpha_{1}$ and $\alpha_{2}$. We restrict the metrics to be diagonal ones and study a class of solutions with exponential time dependence of three scale factors, governed by three non-coinciding Hubble-like parameters: $H \neq 0, h_{1}$ and $h_{2}$, obeying $m H+k_{1} h_{1}+k_{2} h_{2} \neq 0$ and corresponding to factor spaces of dimensions $m>1, k_{1}>1$ and $k_{2}>1$, respectively ( $\left.D=1+m+k_{1}+k_{2}\right)$. We analyse two cases: i) $m<k_{1}<k_{2}$ and ii) $1<k_{1}=k_{2}=k, k \neq m$. We show that in both cases the solutions exist if $\alpha=\alpha_{2} / \alpha_{1}>0$ and $\alpha \Lambda>0$ satisfies certain restrictions, e.g. upper and lower bounds. In case ii) explicit relations for exact solutions are found. In both cases the subclasses of stable and non-stable solutions are singled out. For $m>3$ the case i) contains a subclass of solutions describing an exponential expansion of 3-dimensional subspace with Hubble parameter $H>0$ and zero variation of the effective gravitational constant $G$. The case $H=0$ is also considered.
\end{abstract}

\section{Introduction}

In this paper we consider $D$-dimensional Einstein-GaussBonnet (EGB) model with a $\Lambda$-term. To some extent this model is unique among the other higher-dimensional extensions of General Relativity (GR) with second order in curvature terms. The reason is the following one: the equations of motion for this model are of the second order (in derivatives) like it takes place in the Einstein gravity. It is well known that the so-called Gauss-Bonnet term appeared in (super)string theory as a first order correction (in $\alpha^{\prime}$ ) to the (super)string effective action (e.g. heterotic one) [1-4].

\footnotetext{
a e-mail: ivashchuk@mail.ru (corresponding author)
}

Currently, EGB gravitational model in diverse dimensions and its modifications, see [5-30] and Refs. therein, are rather popular objects for studying in cosmology. They are used for possible explanation of accelerating expansion of the Universe (i.e. solving the dark energy problem), which follow from supernova (type Ia) observational data [31-33]. One may expect that the second order form of the equations of motion for these models will lead us to solutions which are in some sense close to those coming from GR and its higher dimensional extensions (e.g. avoiding the ghosts branches at least).

The $D$-dimensional EGB model is a particular case of the Lovelock model [34]. The equations of motion for the Lovelock model have also at most second order derivatives of the metric (as it takes place in GR). We note that at present there exist several modifications of Einstein and EGB actions which correspond to $F(R), R+f(\mathcal{G}), f(R, \mathcal{G})$ theories (e.g. for $D=4$ ), where $R$ is scalar curvature and $\mathcal{G}$ is GaussBonnet term. These modifications are under intensive studying devoted to cosmological, astrophysical and other applications, see [28-30] and references therein.

In this paper we restrict ourselves to diagonal metrics and study (mainly) a class of cosmological solutions with exponential time dependence of three scale factors, governed by three non-coinciding Hubble-like parameters: $H \neq 0, h_{1}$ and $h_{2}$, corresponding to factor spaces of dimensions $m>1$, $k_{1}>1$ and $k_{2}>1$, respectively, with a restriction imposed: $S_{1}=m H+k_{1} h_{1}+k_{2} h_{2} \neq 0$, and $D=1+m+k_{1}+k_{2}$. This restriction forbids the solutions with constant volume factor. We note that in generic anisotropic case with Hubblelike parameters $h_{1}, \ldots, h_{n}$ obeying $S_{1}=\sum_{i=1}^{n} h_{i} \neq 0$ ( $n=D-1)$ the number of different real numbers among $h_{1}, \ldots, h_{n}$ should not exceed 3 [25].

Here we study two cases: i) $m<k_{1}<k_{2}$ and ii) $1<k_{1}=k_{2}=k, k \neq m$. We show that in both cases the solutions exist only if $\alpha=\alpha_{2} / \alpha_{1}>0, \Lambda>0$ and 
$\Lambda$ obeys certain restrictions, e.g. inequalities of the form: $0<\lambda_{*}\left(m, k_{1}, k_{2}\right)<\Lambda \alpha<\lambda_{* *}\left(m, k_{1}, k_{2}\right)$. We note that in superstring inspired models $\alpha$ is positive and corresponds to Regge slope parameter $\alpha^{\prime}$ which is inverse proportional to the tension of the (super)string; non-zero $\Lambda$-terms appear for non-critical superstrings.

The solutions under consideration are reduced to solutions of polynomial master equation of fourth order or less, which may be solved in radicals for all $m>1, k_{1}>1$ and $k_{2}>1$. In the case ii) $1<k_{1}=k_{2}=k, k \neq m$ we present explicit exact solutions for Hubble-like parameters. Here we use our previous results from refs. [23,25] in studying the stability of the solutions under consideration. In Sect. 5 we single out (for both cases i) and ii)) the subclasses of stable and non-stable solutions. In Sect. 6 we present as an example a subclass of solutions (for the case i)) describing an exponential expansion of 3-dimensional subspace with Hubble parameter $H>0$ and zero variation of the effective gravitational constant $G$ (in Jordan frame) which was obtained in Ref. [26] for fixed value of $\Lambda$ (depending upon $m, k_{1}, k_{2}$ and $\alpha>0)$.

We note that earlier Ref. [27] was dealing with exponential cosmological solutions in the EGB model (with a $\Lambda$-term) with two non-coinciding Hubble-like parameters $H>0$ and $h$ obeying $S_{1}=m H+l h_{1} \neq 0$ and corresponding to $m$ and $l$-dimensional factor spaces $(m>2, l>2)$. In this case there were two sets of solutions obeying: a) $\alpha>0$, $\Lambda<\alpha^{-1} \lambda_{+}(m, l)$ and b) $\alpha<0, \Lambda>|\alpha|^{-1} \lambda_{-}(m, l)$, with $\lambda_{ \pm}(m, l)>0$. Thus, the case of two (non-coinciding) Hubble-like parameters from Ref. [27] drastically differs from the case of three (non-coinciding) Hubble-like parameters which is studied in this paper.

\section{The cosmological model}

The action of the model reads

$$
S=\int_{M} d^{D} z \sqrt{|g|}\left\{\alpha_{1}(R[g]-2 \Lambda)+\alpha_{2} \mathcal{L}_{2}[g]\right\}
$$

where $g=g_{M N} d z^{M} \otimes d z^{N}$ is the metric defined on the manifold $M, \operatorname{dim} M=D,|g|=\left|\operatorname{det}\left(g_{M N}\right)\right|, \Lambda$ is the cosmological term, $R[g]$ is scalar curvature,

$$
\mathcal{L}_{2}[g]=R_{M N P Q} R^{M N P Q}-4 R_{M N} R^{M N}+R^{2}
$$

is the standard Gauss-Bonnet term and $\alpha_{1}, \alpha_{2}$ are nonzero constants.

We consider the manifold

$M=\mathbb{R} \times M_{1} \times \ldots \times M_{n}$ with the metric

$g=-d t \otimes d t+\sum_{i=1}^{n} B_{i} e^{2 v^{i} t} d y^{i} \otimes d y^{i}$,

where $B_{i}>0$ are arbitrary constants, $i=1, \ldots, n$, and $M_{1}, \ldots, M_{n}$ are one-dimensional manifolds (either $\mathbb{R}$ or $S^{1}$ ) and $n>3$.

The equations of motion for the action (2.1) give us the set of polynomial equations [23]

$$
\begin{aligned}
& E=G_{i j} v^{i} v^{j}+2 \Lambda-\alpha G_{i j k l} v^{i} v^{j} v^{k} v^{l}=0 \\
& Y_{i}=\left[2 G_{i j} v^{j}-\frac{4}{3} \alpha G_{i j k l} v^{j} v^{k} v^{l}\right] \sum_{i=1}^{n} v^{i}-\frac{2}{3} G_{i j} v^{i} v^{j}+\frac{8}{3} \Lambda=0
\end{aligned}
$$

$i=1, \ldots, n$, where $\alpha=\alpha_{2} / \alpha_{1}$. Here

$G_{i j}=\delta_{i j}-1, \quad G_{i j k l}=G_{i j} G_{i k} G_{i l} G_{j k} G_{j l} G_{k l}$

are, respectively, the components of two metrics on $\mathbb{R}^{n}$ $[16,17]$. The first one is a 2-metric and the second one is a Finslerian 4-metric. For $n>3$ we get a set of forth-order polynomial equations.

We note that for $\Lambda=0$ and $n>3$ the set of equations (2.4) and (2.5) has an isotropic solution $v^{1}=\cdots=v^{n}=H$ only if $\alpha<0$ [16,17]. This solution was generalized in [19] to the case $\Lambda \neq 0$.

It was shown in $[16,17]$ that there are no more than three different numbers among $v^{1}, \ldots, v^{n}$ when $\Lambda=0$. This is valid also for $\Lambda \neq 0$ if $\sum_{i=1}^{n} v^{i} \neq 0$ [25].

Here we consider a class of solutions to the set of equations (2.4), (2.5) of the following form:

$v=(\overbrace{H, \ldots, H}^{m}, \overbrace{h_{1}, \ldots, h_{1}}^{k_{1}}, \overbrace{h_{2}, \ldots, h_{2}}^{k_{2}})$,

where $H$ is the Hubble-like parameter corresponding to an $m$ dimensional factor space with $m>1, h_{1}$ is the Hubble-like parameter corresponding to an $k_{1}$-dimensional factor space with $k_{1}>1$ and $h_{2}$ is the Hubble-like parameter corresponding to an $k_{2}$-dimensional factor space with $k_{2}>1$. In Sect. 6 we split the $m$-dimensional factor space for $m>3$ into the product of two subspaces of dimensions 3 and $m-3$, respectively. The first one is identified with "our" $3 d$ space while the second one is considered as a subspace of $\left(m-3+k_{1}+k_{2}\right)$ dimensional internal space.

Remark For $H>0$ "our" 3d space expands (isotropically) with Hubble parameter $H$ and the $(m-3)$-dimensional part of internal space $(m>3)$ expands (isotropically) with the same Hubble parameter $\mathrm{H}$ too. Moreover, we may deal with Hubble-like parameters decribing the internal subspaces which obey $h_{1}>H$ or $h_{2}>H$ (see Sect. 6). To avoid possible questions with the separation of subspaces, we consider for physical applications (in our epoch) the internal space to be compact, i.e. we put in (2.2) $M_{4}=\cdots=M_{n}=S^{1}$ 
and we set the internal scale factors corresponding to the present time $t_{0}: a_{j}\left(t_{0}\right)=\left(B_{k}\right)^{1 / 2} \exp \left(v^{j} t_{0}\right), k=4, \ldots, n$, (see (2.3)) to be small enough in comparison with the scale factor of "our" space for $t=t_{0}: a\left(t_{0}\right)=B^{1 / 2} \exp \left(H t_{0}\right)$, where $B_{1}=B_{2}=B_{3}=B>0$.

We consider the ansatz (2.7) with three Hubble-like parameters $H, h_{1}$ and $h_{2}$ which obey the following restrictions:

$$
\begin{aligned}
& H \neq h_{1}, \quad H \neq h_{2}, \quad h_{1} \neq h_{2}, \\
& S_{1}=m H+k_{1} h_{1}+k_{2} h_{2} \neq 0 .
\end{aligned}
$$

In Ref. [26] the set of $(n+1)$ polynomial equations (2.4), (2.5) under ansatz (2.7) and restrictions (2.8) imposed was reduced to a set of three polynomial equations (of fourth, second and first orders, respectively)

$E=0$,

$Q=-\frac{1}{2 \alpha}$,

$L=H+h_{1}+h_{2}-S_{1}=0$.

where $E$ is defined in (2.4) and

$$
\begin{aligned}
Q= & Q_{h_{1} h_{2}}=S_{1}^{2}-S_{2}-2 S_{1}\left(h_{1}+h_{2}\right) \\
& +2\left(h_{1}^{2}+h_{1} h_{2}+h_{2}^{2}\right),
\end{aligned}
$$

where here and in what follows

$S_{k}=\sum_{i=1}^{n}\left(v^{i}\right)^{k}$.

This reduction is a special case of a more general prescription (Chirkov-Pavluchenko-Toporensky trick) from Ref. [20].

Moreover, it was shown in Ref. [26] that the following relations take place

$$
\begin{aligned}
Q_{h_{i} h_{j}}= & S_{1}^{2}-S_{2}-2 S_{1}\left(h_{i}+h_{j}\right) \\
& +2\left(h_{i}^{2}+h_{i} h_{j}+h_{j}^{2}\right)=-\frac{1}{2 \alpha},
\end{aligned}
$$

where $i \neq j ; i, j=0,1,2$ and $h_{0}=H$.

Due to (2.8) the case $H=h_{1}=h_{2}=0$ is excluded. First, we put

$H \neq 0$.

Let us denote

$x_{1}=h_{1} / H, \quad x_{2}=h_{2} / H$.

Then restrictions (2.8) read

$x_{1} \neq 1, \quad x_{2} \neq 1, \quad x_{1} \neq x_{2}, \quad m+k_{1} x_{1}+k_{2} x_{2} \neq 0$.

Equation (2.11) in $x$-variables reads

$m-1+\left(k_{1}-1\right) x_{1}+\left(k_{2}-1\right) x_{2}=0$.
Here we should exclude from our consideration the case

$m=k_{1}=k_{2}$.

Indeed, for $m=k_{1}=k_{2}>1$ we get from restriction (2.17): $1+x_{1}+x_{2} \neq 0$, while $(2.18)$ gives us the relation $1+x_{1}+x_{2}=$ 0 , which is incompatible with the previous one.

We get from (2.10) and (2.12) that

$2 \alpha \mathcal{P} H^{2}=-1$

where

$$
\begin{aligned}
\mathcal{P}= & \mathcal{P}\left(x_{1}, x_{2}\right)=\mathcal{P}\left(x_{1}, x_{2}, m, k_{1}, k_{2}\right) \\
= & \left(m+k_{1} x_{1}+k_{2} x_{2}\right)^{2}-\left(m+k_{1} x_{1}^{2}+k_{2} x_{2}^{2}\right) \\
& -2\left(m+k_{1} x_{1}+k_{2} x_{2}\right)\left(x_{1}+x_{2}\right)+2\left(x_{1}^{2}+x_{1} x_{2}+x_{2}^{2}\right) .
\end{aligned}
$$

We note that relation (2.20) is obeyed for $\alpha \mathcal{P}<0$. Let us prove that

$\mathcal{P}<0$

Indeed, using relation (2.18), or $m+k_{1} x_{1}+k_{2} x_{2}=1+x_{1}+x_{2}$, we get

$$
\begin{aligned}
\mathcal{P}= & \left(1+x_{1}+x_{2}\right)^{2}-\left(m+k_{1} x_{1}^{2}+k_{2} x_{2}^{2}\right) \\
& -2\left(1+x_{1}+x_{2}\right)\left(x_{1}+x_{2}\right)+2\left(x_{1}^{2}+x_{1} x_{2}+x_{2}^{2}\right) \\
= & 1-m+\left(1-k_{1}\right) x_{1}^{2}+\left(1-k_{2}\right) x_{2}^{2}<0
\end{aligned}
$$

for $m>1, k_{1}>1, k_{2}>1$.

Hence, the solutions under consideration take place only if

$\alpha>0$.

The calculations gives us the following relation for the vector $v$ from (2.7)

$G_{i j} v^{i} v^{j}=m H^{2}+k_{1} h_{1}^{2}+k_{2} h_{2}^{2}-\left(m H+k_{1} h_{1}+k_{2} h_{2}\right)^{2}$

and

$$
\begin{aligned}
G_{i j k l} v^{i} v^{j} v^{k} v^{l}= & m(m-1)(m-2)(m-3) H^{4} \\
& +4 m(m-1)(m-2) H^{3}\left(k_{1} h_{1}+k_{2} h_{2}\right) \\
& +6 m(m-1) H^{2}\left[k_{1}\left(k_{1}-1\right) h_{1}^{2}\right. \\
& \left.+2 k_{1} k_{2} h_{1} h_{2}+k_{2}\left(k_{2}-1\right) h_{2}^{2}\right] \\
& +4 m H\left[k_{1}\left(k_{1}-1\right)\left(k_{1}-2\right) h_{1}^{3}+3 k_{1}\left(k_{1}-1\right) k_{2} h_{1}^{2} h_{2}\right. \\
& \left.+3 k_{1} k_{2}\left(k_{2}-1\right) h_{1} h_{2}^{2}+k_{2}\left(k_{2}-1\right)\left(k_{2}-2\right) h_{2}^{3}\right] \\
& +k_{1}\left(k_{1}-1\right)\left(k_{1}-2\right)\left(k_{1}-3\right) h_{1}^{4} \\
& +4 k_{1}\left(k_{1}-1\right)\left(k_{1}-2\right) k_{2} h_{1}^{3} h_{2} \\
& +6 k_{1}\left(k_{1}-1\right) k_{2}\left(k_{2}-1\right) h_{1}^{2} h_{2}^{2} \\
& +4 k_{1} k_{2}\left(k_{2}-1\right)\left(k_{2}-2\right) h_{1} h_{2}^{3} \\
& +k_{2}\left(k_{2}-1\right)\left(k_{2}-2\right)\left(k_{2}-3\right) h_{2}^{4} .
\end{aligned}
$$


This may be obtained by using the relation from Ref. [17]

$$
G_{i j k l} v^{i} v^{j} v^{k} v^{l}=S_{1}^{4}-6 S_{1}^{2} S_{2}+3 S_{2}^{2}+8 S_{1} S_{3}-6 S_{4} \text {. }
$$

Due to (2.4), (2.25) and (2.26), the Eq. (2.9) reads

$$
\begin{aligned}
2 \Lambda & =-G_{i j} v^{i} v^{j}+\alpha G_{i j k l} v^{i} v^{j} v^{k} v^{l} \\
& =H^{2} V_{1}+\alpha H^{4} V_{2},
\end{aligned}
$$

where

$$
\begin{aligned}
V_{1} & =V_{1}\left(x_{1}, x_{2}\right)=V_{1}\left(x_{1}, x_{2}, m, k_{1}, k_{2}\right) \\
& =-m-k_{1} x_{1}^{2}-k_{2} x_{2}^{2}+\left(m+k_{1} x_{1}+k_{2} x_{2}\right)^{2}
\end{aligned}
$$

and

$$
\begin{aligned}
V_{2}= & V_{2}\left(x_{1}, x_{2}\right)=V_{2}\left(x_{1}, x_{2}, m, k_{1}, k_{2}\right) \\
= & {[m]_{4}+4[m]_{3}\left(k_{1} x_{1}+k_{2} x_{2}\right) } \\
& +6[m]_{2}\left(\left[k_{1}\right]_{2} x_{1}^{2}+2 k_{1} k_{2} x_{1} x_{2}+\left[k_{2}\right]_{2} x_{2}^{2}\right) \\
& +4 m\left(\left[k_{1}\right]_{3} x_{1}^{3}+3\left[k_{1}\right]_{2} k_{2} x_{1}^{2} x_{2}\right. \\
& \left.+3 k_{1}\left[k_{2}\right]_{2} x_{1} x_{2}^{2}+\left[k_{2}\right]_{3} x_{2}^{3}\right) \\
& +\left[k_{1}\right]_{4} x_{1}^{4}+4\left[k_{1}\right]_{3} k_{2} x_{1}^{3} x_{2}+6\left[k_{1}\right]_{2}\left[k_{2}\right]_{2} x_{1}^{2} x_{2}^{2} \\
& +4 k_{1}\left[k_{2}\right]_{3} x_{1} x_{2}^{3}+\left[k_{2}\right]_{4} x_{2}^{4} .
\end{aligned}
$$

Here we use the notation $[N]_{k}=N(N-1) \ldots(N-k+1)$.

Using (2.20) we get

$\lambda=\alpha \Lambda=-\frac{V_{1}}{4 \mathcal{P}}+\frac{V_{2}}{8 \mathcal{P}^{2}}$,

or, equivalently,

$V_{2}\left(x_{1}, x_{2}\right)-2 \mathcal{P}\left(x_{1}, x_{2}\right) V_{1}\left(x_{1}, x_{2}\right)-8\left(\mathcal{P}\left(x_{1}, x_{2}\right)\right)^{2} \lambda=0$.

Thus, we are led to polynomial equation in variables $x_{1}, x_{2}$ of fourth order or less (depending upon $\lambda$ ).

We call relations (2.18), (2.32), as a master equations. The set of these equations may solved in radicals. Indeed, solving eq. (2.18)

$x_{2}=x_{2}\left(x_{1}\right)=-\frac{m-1}{k_{2}-1}-\frac{k_{1}-1}{k_{2}-1} x_{1}$

and substituting into eq. (2.32) we obtain another (master) equation in $x_{1}$

$$
\begin{aligned}
& V_{2}\left(x_{1}, x_{2}\left(x_{1}\right)\right)-2 \mathcal{P}\left(x_{1}, x_{2}\left(x_{1}\right)\right) V_{1}\left(x_{1}, x_{2}\left(x_{1}\right)\right) \\
& \quad-8\left(\mathcal{P}\left(x_{1}, x_{2}\left(x_{1}\right)\right)\right)^{2} \lambda=0,
\end{aligned}
$$

which is of fourth order or less depending upon the value of $\lambda$. It may solved in radicals for all $m>1, k_{1}>1$ and $k_{2}>1$. Here we do not try to write the explicit solution for general setup. It seems more effective for any given dimensions $m, k_{1}$ and $k_{2}$ to find the solutions just by using Maple or Mathematica. An example of solution with $k_{1}=k_{2}$ will be considered below.
In what follows we use the identity

$$
\begin{aligned}
& -\left(k_{2}-1\right) \mathcal{P}\left(x_{1}, x_{2}\left(x_{1}\right)\right) \\
& \quad=\left(k_{1}-1\right)\left(k_{1}+k_{2}-2\right) x_{1}^{2} \\
& \quad+2(m-1)\left(k_{1}-1\right) x_{1}+(m-1)\left(m+k_{2}-2\right),
\end{aligned}
$$

following from (2.23) and (2.33).

\section{The case $k_{1} \neq k_{2}$}

Here we put the following restriction $k_{1} \neq k_{2}$. We write relation (2.31) as

$\lambda=f\left(x_{1}\right) \equiv-\frac{V_{1}\left(x_{1}, x_{2}\left(x_{1}\right)\right)}{4 \mathcal{P}\left(x_{1}, x_{2}\left(x_{1}\right)\right)}+\frac{V_{2}\left(x_{1}, x_{2}\left(x_{1}\right)\right)}{8\left(\mathcal{P}\left(x_{1}, x_{2}\left(x_{1}\right)\right)\right)^{2}}$.

Using relation (2.33) we rewrite the restrictions (2.17) (respectively) as follows

$x_{1} \neq X_{1}, \quad x_{1} \neq X_{2}, \quad x_{1} \neq X_{3}, \quad x_{1} \neq X_{4}$,

where

$X_{1}=1$,

$X_{2}=-\frac{m+k_{2}-2}{k_{1}-1}$,

$X_{3}=-\frac{m-1}{k_{1}+k_{2}-2}$,

$X_{4}=\frac{m-k_{2}}{k_{2}-k_{1}}$.

\subsection{Extremum points}

The calculations give us

$\frac{d f}{d x_{1}}=\frac{C\left(m, k_{1}, k_{2}\right)\left(x_{1}-X_{1}\right)\left(x_{1}-X_{2}\right)\left(x_{1}-X_{3}\right)\left(x_{1}-X_{4}\right)}{\left(-\left(k_{2}-1\right) \mathcal{P}\left(x_{1}, x_{2}\left(x_{1}\right)\right)\right)^{3}}$,

where

$C\left(m, k_{1}, k_{2}\right)=(m-1)\left(k_{1}-1\right)^{2}\left(k_{2}-k_{1}\right)\left(k_{1}+k_{2}-2\right)$

and $X_{1}, X_{2}, X_{3}, X_{4}$ are defined in (3.3)-(3.6). Thus, the points of extremum of the function $f\left(x_{1}\right)$ are excluded from our consideration due to restrictions (2.8).

For the values $\lambda_{i}=f\left(X_{i}\right), i=1,2,3,4$, we get

$$
\begin{aligned}
& \lambda_{1}=\lambda_{1}\left(m, k_{1}, k_{2}\right)=\frac{u\left(k_{2}, m+k_{1}\right)}{8\left(m+k_{1}+k_{2}-3\right)\left(m+k_{1}-2\right)\left(k_{2}-1\right)}, \\
& \lambda_{2}=\lambda_{2}\left(m, k_{1}, k_{2}\right)=\frac{u\left(k_{1}, m+k_{2}\right)}{8\left(m+k_{1}+k_{2}-3\right)\left(m+k_{2}-2\right)\left(k_{1}-1\right)}, \\
& \lambda_{3}=\lambda_{3}\left(m, k_{1}, k_{2}\right)=\frac{u\left(m, k_{1}+k_{2}\right)}{8(m-1)\left(k_{1}+k_{2}-2\right)\left(m+k_{1}+k_{2}-3\right)}, \\
& \lambda_{4}=\lambda_{4}\left(m, k_{1}, k_{2}\right)=\frac{v\left(m, k_{1}, k_{2}\right)}{8 w\left(m, k_{1}, k_{2}\right)},
\end{aligned}
$$


where

$$
\begin{aligned}
u(m, l) & =l m^{2}+\left(l^{2}-8 l+8\right) m+l(l-1), \\
v(m, l, k) & =(k+l) m^{2}+(m+l) k^{2}+(m+k) l^{2}-6 m l k,
\end{aligned}
$$$$
w(m, l, k)=(k+l-2) m^{2}+(m+l-2) k^{2}+(m+k-2) l^{2}
$$

$$
+2 m l+2 m k+2 l k-6 m l k \text {. }
$$

We note that

$\lambda_{i}=\lambda_{i}\left(m, k_{1}, k_{2}\right)>0$

for all $m>1, k_{1}>1, k_{2}>1, i=1,2,3,4$.

For $i=1,2,3$ this relation follows from the

$u(m, l)>0$

for $m>1$ and $l>1$. Indeed, for $m \geq 4, l \geq 4$ we get $u(m, l)=m l(m+l-8)+8 m+l^{2}-l>0$ and $u(4,3)=26$, $u(3,4)=24, u(3,3)=12, u(3,2)=8, u(2,3)=4$, $u(2,2)=2$. For $i=4$ the relation (3.16) follows from the inequalities

$v(m, l, k)>0$,

$w(m, l, k)>0$,

which are valid for natural numbers $m, l, k$ obeying: $m>1$, $l>1, k>1$ and either $m \neq l$, or $m \neq k$, or $l \neq k$. This is proved in "Appendix".

We also note that the following symmetry identities take place for the functions $\lambda_{i}\left(m, k_{1}, k_{2}\right), i=1,2,3$,

$\lambda_{1}\left(m, k_{1}, k_{2}\right)=\lambda_{2}\left(m, k_{2}, k_{1}\right)=\lambda_{3}\left(k_{2}, m, k_{1}\right)$,

$\lambda_{3}\left(m, k_{1}, k_{2}\right)=\lambda_{3}\left(m, k_{2}, k_{1}\right)$.

The function $\lambda_{4}\left(m, k_{1}, k_{2}\right)$ is symmetric with respect to variables since the functions $v\left(m, k_{1}, k_{2}\right)$ and $w\left(m, k_{1}, k_{2}\right)$ are symmetric.

For $x_{1} \rightarrow \pm \infty$ we get

$$
\begin{aligned}
\lambda_{\infty} & =\lim _{x_{1} \rightarrow \infty} f\left(x_{1}\right) \\
& =\frac{\left(k_{1}+k_{2}-6\right) k_{1} k_{2}+k_{1}^{2}+k_{2}^{2}+k_{1}+k_{2}}{8\left(k_{1}-1\right)\left(k_{2}-1\right)\left(k_{1}+k_{2}-2\right)} .
\end{aligned}
$$

It may be readily verified that

$\lambda_{\infty}=\lambda_{\infty}\left(k_{1}, k_{2}\right)=\lambda_{\infty}\left(k_{2}, k_{1}\right)>0$,

for all $k_{1}>1$ and $k_{2}>1$. Indeed, $\left(k_{1}+k_{2}-6\right) k_{1} k_{2}+k_{1}^{2}+$ $k_{2}^{2}+k_{1}+k_{2}=\left(k_{1}+k_{2}-4\right) k_{1} k_{2}+\left(k_{1}-k_{2}\right)^{2}+k_{1}+k_{2}>0$ for $k_{1} \geq 2$ and $k_{2} \geq 2$.

The points of extremum obey the following relations

$$
\begin{aligned}
& X_{2}-X_{1}=-\frac{m+k_{1}+k_{2}-3}{k_{1}-1}, \\
& X_{3}-X_{1}=-\frac{m+k_{1}+k_{2}-3}{k_{1}+k_{2}-2},
\end{aligned}
$$

$$
\begin{aligned}
& X_{3}-X_{2}=\frac{\left(m+k_{1}+k_{2}-3\right)\left(k_{2}-1\right)}{\left(k_{1}+k_{2}-2\right)\left(k_{1}-1\right)}, \\
& X_{4}-X_{1}=-\frac{m+k_{1}-2 k_{2}}{k_{1}-k_{2}}, \\
& X_{4}-X_{2}=\frac{\left(m-2 k_{1}+k_{2}\right)\left(k_{2}-1\right)}{\left(k_{1}-1\right)\left(k_{2}-k_{1}\right)}, \\
& X_{4}-X_{3}=\frac{\left(2 m-k_{1}-k_{2}\right)\left(k_{2}-1\right)}{\left(k_{1}+k_{2}-2\right)\left(k_{2}-k_{1}\right)} .
\end{aligned}
$$

It follows from definitions of $X_{i}$ and (3.24), (3.25), (3.26) that

$X_{2}<X_{3}<0<X_{1}=1$

for all $m>1, k_{1}>1$ and $k_{2}>1$.

The corresponding relations for $\lambda_{i}-\lambda_{j}$ have the following form

$\lambda_{2}-\lambda_{1}=\frac{(m-1)\left(k_{2}-k_{1}\right)\left(m+k_{1}+k_{2}-3\right)}{4\left(k_{1}-1\right)\left(k_{2}-1\right)\left(m+k_{1}-2\right)\left(m+k_{2}-2\right)}$

$\lambda_{3}-\lambda_{1}=\frac{\left(k_{1}-1\right)\left(k_{2}-m\right)\left(m+k_{1}+k_{2}-3\right)}{4(m-1)\left(k_{2}-1\right)\left(m+k_{1}-2\right)\left(k_{1}+k_{2}-2\right)}$,

$\lambda_{3}-\lambda_{2}=\frac{\left(k_{2}-1\right)\left(k_{1}-m\right)\left(m+k_{1}+k_{2}-3\right)}{4(m-1)\left(k_{1}-1\right)\left(m+k_{2}-2\right)\left(k_{1}+k_{2}-2\right)}$,

$\lambda_{4}-\lambda_{1}=\frac{(m-1)\left(k_{1}-1\right)\left(2 k_{2}-k_{1}-m\right)^{3}}{4\left(m+k_{1}-2\right)\left(k_{2}-1\right)\left(m+k_{1}+k_{2}-3\right) w}$,

$\lambda_{4}-\lambda_{2}=\frac{(m-1)\left(k_{2}-1\right)\left(2 k_{1}-m-k_{2}\right)^{3}}{4\left(m+k_{2}-2\right)\left(k_{1}-1\right)\left(m+k_{1}+k_{2}-3\right) w}$,

$\lambda_{4}-\lambda_{3}=\frac{\left(k_{1}-1\right)\left(k_{2}-1\right)\left(2 m-k_{1}-k_{2}\right)^{3}}{4\left(k_{1}+k_{2}-2\right)(m-1)\left(m+k_{1}+k_{2}-3\right) w}$,

where $w=w\left(m, k_{1}, k_{2}\right)$ is defined in (3.15).

Here and in what follows up to the Sect. 4 we put that

$1<m<k_{1}<k_{2}$.

Using (3.31), (3.33) and (3.37) we get

$0<\lambda_{1}<\lambda_{2}<\lambda_{3}$.

Analogously, using (3.34), (3.36) and (3.37) we get

$0<\lambda_{1}<\lambda_{4}<\lambda_{3}$.

It follows from (3.28), (3.35) and (3.37) that

$\left(A_{+}\right) \quad X_{4}<X_{2}, \quad \lambda_{4}>\lambda_{2}, \quad$ for $2 k_{1}-m-k_{2}>0$,

$\left(A_{-}\right) \quad X_{4}>X_{2}, \quad \lambda_{4}<\lambda_{2}, \quad$ for $2 k_{1}-m-k_{2}<0$,

and

(A $\left.A_{0}\right) \quad X_{4}=X_{2}, \quad \lambda_{4}=\lambda_{2}, \quad$ for $2 k_{1}-m-k_{2}=0$.

The graphical representations of the function $\lambda=f\left(x_{1}\right)$ for $\left(m, k_{1}, k_{2}\right)=(4,6,7),(4,5,7),(4,5,6)$ are given at Figs. 1, 2 and 3, respectively. These three sets obey the inequalities (3.40), (3.41) and (3.42), respectively. 
For $\lambda_{i}-\lambda_{\infty}$ we obtain

$$
\begin{aligned}
& \lambda_{1}-\lambda_{\infty}=\frac{(m-1) z_{1}}{4\left(k_{1}-1\right)\left(k_{1}+k_{2}-2\right)\left(m+k_{1}-2\right)\left(m+k_{1}+k_{2}-3\right)}, \\
& \lambda_{2}-\lambda_{\infty}=\frac{(m-1) z_{2}}{4\left(k_{2}-1\right)\left(k_{1}+k_{2}-2\right)\left(m+k_{2}-2\right)\left(m+k_{1}+k_{2}-3\right)}, \\
& \lambda_{3}-\lambda_{\infty}=\frac{z_{3}}{4(m-1)\left(k_{1}-1\right)\left(k_{2}-1\right)\left(k_{1}+k_{2}-2\right)\left(m+k_{1}+k_{2}-3\right)}, \\
& \lambda_{4}-\lambda_{\infty}=\frac{(m-1) z_{4}}{4\left(k_{1}-1\right)\left(k_{2}-1\right)\left(k_{1}+k_{2}-2\right) w},
\end{aligned}
$$

where

$$
\begin{aligned}
z_{1}= & \left(2 k_{1}-k_{2}-1\right) m-2 k_{1} k_{2}-4 k_{1}+5 k_{2}-k_{2}^{2}+2 k_{1}^{2}, \\
z_{2}= & \left(2 k_{2}-k_{1}-1\right) m-2 k_{1} k_{2}-4 k_{2}+5 k_{1}-k_{1}^{2}+2 k_{2}^{2}, \\
z_{3}= & -\left(k_{1}-k_{2}\right)^{2} m^{2}-\left(\left(k_{1}^{2}-6 k_{1}+6\right) k_{1}+\left(k_{2}^{2}-6 k_{2}+6\right) k_{2}\right. \\
& \left.+\left(k_{1}+k_{2}\right) k_{1} k_{2}-4\right) m-2\left(k_{1}+k_{2}\right) \\
& +\left(12-6 k_{1}-6 k_{2}+\left(k_{1}+k_{2}\right)^{2}\right) k_{1} k_{2}, \\
z_{4}= & -\left(k_{2}-k_{1}\right)^{2}\left(\left(k_{1}+k_{2}-2\right) m+\left(k_{1}-k_{2}\right)^{2}+k_{1}-2 k_{1} k_{2}+k_{2}\right) .
\end{aligned}
$$

It follows from (3.43), (3.45) and inequalities $z_{1}<0$, $z_{3}>0$, proved in Appendix, that

$\lambda_{1}<\lambda_{\infty}<\lambda_{3}$.

For our restriction (3.37) we obtain from (3.8)

$C\left(m, k_{1}, k_{2}\right)>0$.

In what follows we use the relation (3.7) and inequalities (2.22) and (3.52).

We find that (in all cases) the function $\lambda=f\left(x_{1}\right)$ is monotonically increasing in the interval $\left(X_{1}=1,+\infty\right)$ from $\lambda_{1}$ to $\lambda_{\infty}$ and it is monotonically decreasing in the interval $\left(X_{3}, X_{1}\right)$ from $\lambda_{3}$ to $\lambda_{1}$.

In the case $\left(A_{+}\right)$the function $\lambda=f\left(x_{1}\right)$ is monotonically increasing in the intervals $\left(-\infty, X_{4}\right)$ and $\left(X_{2}, X_{3}\right)$ from $\lambda_{\infty}$ to $\lambda_{4}$ and from $\lambda_{2}$ to $\lambda_{3}$, respectively, while it is monotonically decreasing in the interval $\left(X_{4}, X_{2}\right)$ from $\lambda_{4}$ to $\lambda_{2}$ (see Fig. 1). In this case the points $X_{1}$ and $X_{2}$ are points of local minimum and points $X_{3}$ and $X_{4}$ are points of local maximum.

For the case $\left(A_{-}\right)$the function $\lambda=f\left(x_{1}\right)$ is monotonically increasing in the intervals $\left(-\infty, X_{2}\right)$ and $\left(X_{4}, X_{3}\right)$ from $\lambda_{\infty}$ to $\lambda_{2}$ and from $\lambda_{4}$ to $\lambda_{3}$, respectively, while it is monotonically decreasing in the interval $\left(X_{2}, X_{4}\right)$ from $\lambda_{2}$ to $\lambda_{4}$ (see Fig. 2). The points $X_{1}$ and $X_{4}$ are points of local minimum and points $X_{2}$ and $X_{3}$ are points of local maximum. In this case $\lambda_{2}>\lambda_{\infty}$.

In the case $\left(A_{0}\right)$ the function $\lambda=f\left(x_{1}\right)$ is monotonically increasing in the intervals $\left(-\infty, X_{3}\right)$ from $\lambda_{\infty}$ to $\lambda_{3}$, respectively (see Fig. 3). For this case the point $X_{1}$ is the point of local minimum, the point $X_{3}$ is a point of local maximum and the point $X_{2}=X_{4}$ is a point of inflection.

Using the inequalities (3.38), (3.39) and (3.51) we get from the behaviour of the function $f\left(x_{1}\right)$ mentioned above that $X_{3}$ is the point of absolute maximum and $X_{1}$ is the point of absolute minimum, i.e.

$\lambda_{1} \leq \lambda=f\left(x_{1}\right) \leq \lambda_{3}$

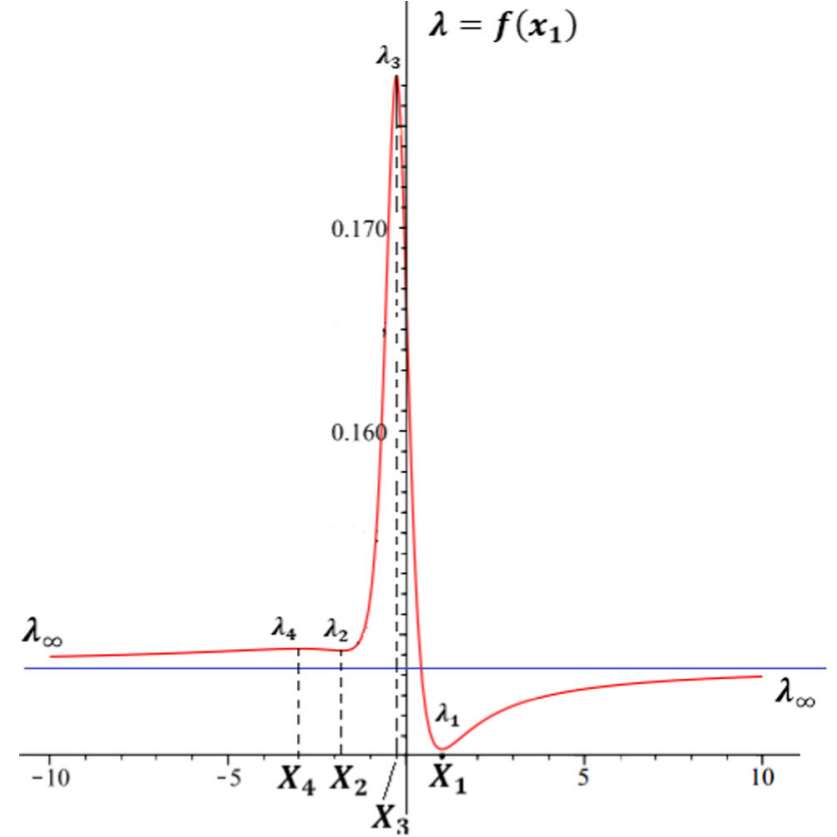

Fig. 1 The function $\lambda=f\left(x_{1}\right)$ for $m=4, k_{1}=6, k_{2}=7$

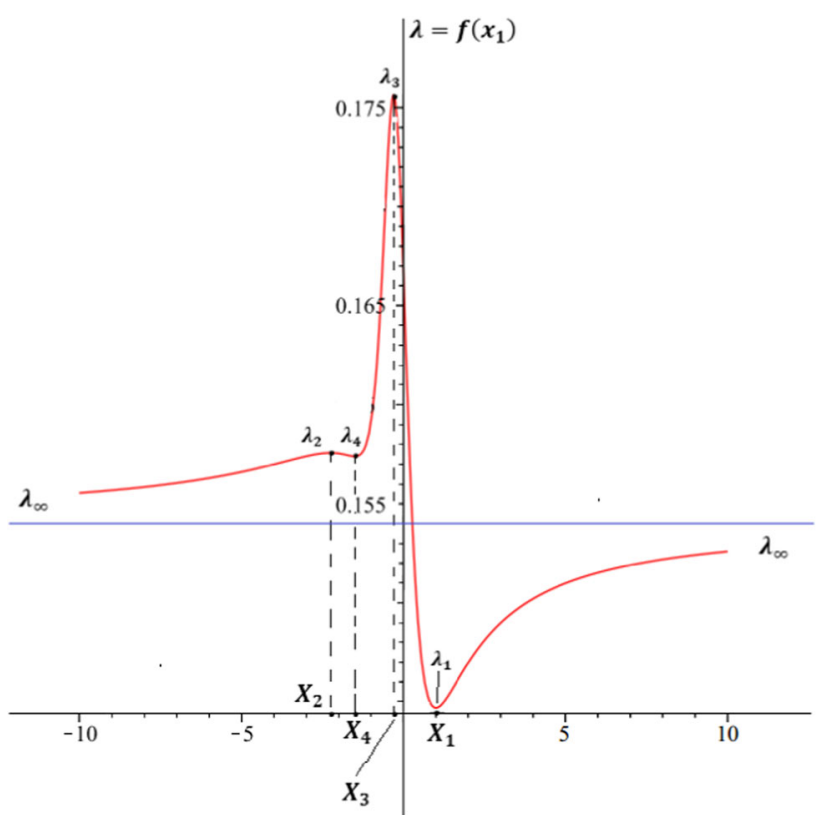

Fig. 2 The function $\lambda=f\left(x_{1}\right)$ for $m=4, k_{1}=5, k_{2}=7$

for all $x_{1} \in \mathbb{R}$. Due to (3.2) the points $X_{1}, X_{2}, X_{3}, X_{4}$ are forbidden for our consideration. We get

$\lambda_{1}<\lambda=f\left(x_{1}\right)<\lambda_{3}$

for all $x_{1} \neq X_{1}, X_{2}, X_{3}, X_{4}$. Let us denote the set of definition of the fuction $f$ for our consideration $(-\infty, \infty)_{*} \equiv$ $\left\{x \mid x \in \mathbb{R}, x \neq X_{1}, X_{2}, X_{3}, X_{4}\right\}$. Since the function $f\left(x_{1}\right)$ is continuous one the image of the function $f$ (due to interme- 


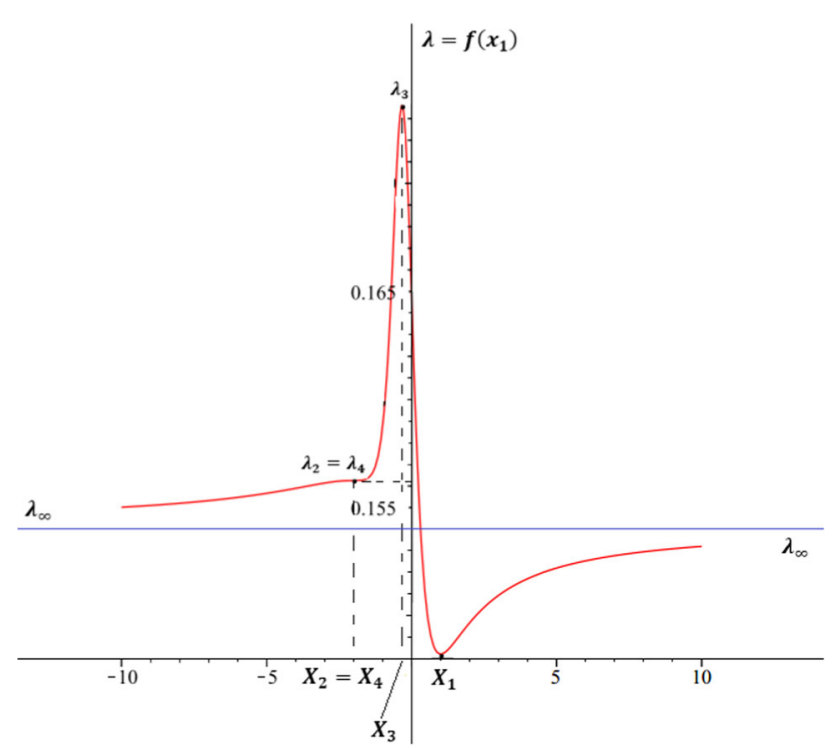

Fig. 3 The function $\lambda=f\left(x_{1}\right)$ for $m=4, k_{1}=5, k_{2}=6$

diate value theorem) is

$f\left((-\infty, \infty)_{*}\right)=\left(\lambda_{1}, \lambda_{3}\right)$.

Thus, we a led the following proposition.

Proposition 1 The solutions to equations (2.4), (2.5) for ansatz (2.7) with $1<m<k_{1}<k_{2}$ obeying the inequalities $H \neq 0, H \neq h_{1}, H \neq h_{2}, h_{1} \neq h_{2}$ and $S_{1}=$ $m H+k_{1} h_{1}+k_{2} h_{2} \neq 0$ do exist if and only if $\alpha>0$ and

$0<\lambda_{1}<\alpha \Lambda<\lambda_{3}$

where $\lambda_{1}$ and $\lambda_{3}$ are defined in (3.9) and (3.11), respectively. In this case $x_{1}=h_{1} / H \neq X_{1}, X_{2}, X_{3}, X_{4}$ (see (3.3), (3.4), (3.5), (3.6)), $x_{2}=h_{2} / H=x_{2}\left(x_{1}\right)$ is given by (2.33), $x_{1}$ obeys the polynomial master equation (2.34) (offourth order or less) and $H^{2}$ is given by (2.20) and (2.21).

The case $H=0$. It may verified that in the case $H=0$ the solutions under consideration take place only if $\alpha>0$, and

$\alpha \Lambda=\lambda_{\infty}\left(k_{1}, k_{2}\right)=\frac{\left(k_{1}+k_{2}-6\right) k_{1} k_{2}+k_{1}^{2}+k_{2}^{2}+k_{1}+k_{2}}{8\left(k_{1}-1\right)\left(k_{2}-1\right)\left(k_{1}+k_{2}-2\right)}>0$,

where $k_{1} \neq k_{2}$. Indeed (2.11) is equivalent to $\left(k_{1}-1\right) h_{1}+$ $\left(k_{2}-1\right) h_{2}=0$, while $(2.10)$ reads as $\left(k_{1}-1\right)\left(h_{1}\right)^{2}+\left(k_{2}-\right.$ 1) $\left(h_{2}\right)^{2}=1 /(2 \alpha)$. These relations imply $\alpha>0$ and

$h_{1}= \pm\left(\frac{k_{2}-1}{2 \alpha\left(k_{1}-1\right)\left(k_{1}+k_{2}-2\right)}\right)^{1 / 2}$,

$h_{2}=\mp\left(\frac{k_{1}-1}{2 \alpha\left(k_{2}-1\right)\left(k_{1}+k_{2}-2\right)}\right)^{1 / 2}$.

The substitution of these values of $h_{1}$ and $h_{2}$, and $H=0$ into equation (2.9) gives us (due to (2.25) and (2.26)) relation (3.57).

\section{The case $k_{1}=k_{2}$}

Here we consider the case $m>1, k_{1}=k_{2}=k>1$ and $H \neq 0$. We get from (2.18)

$m-1+(k-1)\left(x_{1}+x_{2}\right)=0$.

In this case relation (2.23) implies

$\mathcal{P}=1-m+(1-k)\left(x_{1}^{2}+x_{2}^{2}\right)$.

The solutions under consideration take place for

$m \neq k$

and $\alpha>0$ (see Sect. 2).

Let us denote

$X \equiv \alpha H^{2}$,

$\alpha>0$. It follows from (2.20)

$X \mathcal{P}=-\frac{1}{2}$.

Due to (4.4) we have

$H=\varepsilon_{0} \sqrt{X / \alpha}, \quad \varepsilon_{0}= \pm 1$.

The substitution of relations (4.1), (4.2) into formulae (2.29), (2.30) gives us

$$
\begin{aligned}
V_{1}= & {[(m-1)(m-k)+\mathcal{P} k(k-1)] /(k-1)^{2}, } \\
V_{2}= & {[-(m-1)(m-k)(m+k-2)(m+2 k-3)} \\
& \left.+3 \mathcal{P}^{2}(k-1)^{2} k\right] /(k-1)^{3} .
\end{aligned}
$$

Using (4.5) we rewrite relation (2.31) as

$2 \lambda=2 \alpha \Lambda=X V_{1}+X^{2} V_{2}$.

This relation may be written as quadratic relation

$A X^{2}+B X+C=0$,

where

$A=(m-1)(m-k)(m+k-2)(m+2 k-3)$,

$B=-(m-1)(m-k)(k-1)$,

$C=-\frac{1}{4} k(k-1)^{2}+2 \lambda(k-1)^{3}$.

Due to (4.3) $A \neq 0$. The discriminant $D=B^{2}-4 A C$ has the folowing form

$D=(m-1)(m-k)(k-1)^{2}(F-8 \lambda f)$,

where

$$
\begin{aligned}
F= & F(m, k)=(m-1)(m-k) \\
& +(m+k-2)(m+2 k-3) k \\
f= & f(m, k)=(m+k-2)(m+2 k-3)(k-1)>0
\end{aligned}
$$


Lemma $F=F(m, k)>0$ for all $m>1, k>1$ and $k \neq m$.

Proof For $m>k$ we have a sum of two positive terms in (4.15) and hence $F>0$ in this case. For $k>m$, we denote $k=m+p, p>0$. We obtain

$$
\begin{aligned}
F= & (m-1)(-p)+(2 m+p-2) \\
& (3 m+2 p-3)(m+p)= \\
= & (m-1)(-p)+(2(m-1)+p) \\
& (3(m-1)+2 p)(m+p)= \\
= & 2 p^{3}+(9 m-7) p^{2}+(m-1)(13 m-7) p \\
& +6 m(m-1)^{2} .
\end{aligned}
$$

Due to $m>1$ and $p>0$ we have a sum of three positive terms in (4.17) and hence $F>0$ for $k>m$.

The solution to eq. (4.10) reads

$X=\left(-B+\bar{\varepsilon}_{1} \sqrt{D}\right) /(2 A), \quad \bar{\varepsilon}_{1}= \pm 1$.

We are seeking real soutions which obey two restrictions

$D>0$,

$X>0$.

Here the case $D=0$ is excluded from the consideration since as it will be shown later it implies either $x_{1}=1$ or $x_{2}=1$, which contradict restrictions (2.17).

The inequality (4.19) may be rewritten as

$\lambda<\lambda_{1}$ for $m>k$,

$\lambda>\lambda_{1}$ for $m<k$,

where

$\lambda_{1}=\lambda_{1}(m, k, k)=F(m, k) /(8 f(m, k))$.

For definition of $\lambda_{1}(m, k, l)$ see (3.9).

The set of two equations (4.1) and (4.2) have the following solutions

$x_{1}=-\left(\varepsilon_{2} \sqrt{E}+m-1\right) /(2 k-2)$,

$x_{2}=-\left(-\varepsilon_{2} \sqrt{E}+m-1\right) /(2 k-2)$,

where $\varepsilon_{2}= \pm 1$ and

$$
\begin{aligned}
E & =-(m-1)(m+2 k-3)-2 \mathcal{P}(k-1) \\
& =(k-1) X^{-1}-(m-1)(m+2 k-3) .
\end{aligned}
$$

Here we put

$$
E>0
$$

since $E=0$ implies the identity $x_{1}=x_{2}$ which is excluded by restrictions (2.17). The relations (4.20) and (4.27) may be written as

$0<X<\frac{k-1}{(m-1)(m+2 k-3)}$.
Now we explain why the case $D=0$ was excluded from our consideration. Let us put $D=0$. Then we get from (4.18)

$$
X=(-B) /(2 A)=(k-1) /(2(m+k-2)
$$$$
\times(m+2 k-3))
$$

and hence

$E=(m+2 k-3)^{2}$,

which implies either $x_{2}=1$ for $\varepsilon_{2}=1$ or $x_{1}=1$ for $\varepsilon_{2}=-1$. But this is forbiden by first two inequalities in (2.17).

Moreover, it is not difficult to verify that relations (4.24), (4.25) and (4.28) imply all four inequalities in (2.17). Indeed, the violation of first two inequalities in (2.17) lead us either to $x_{1}=1$ or $x_{2}=1$ which may be valid only for $E$ from (4.30) and $\varepsilon_{2}=-1$ or $\varepsilon_{2}=1$, respectively. But due to definition (4.26), relation (4.30) implies (4.29) and hence $D=0$, which contradict to relations (4.24), (4.25). The violation of the third inequality gives us $x_{1}=x_{2}$ which imply $E=0$, but this is forbidden by (4.28). Now, let us verify the last inequality in (2.17). In our case it reads

$x_{1}+x_{2} \neq-\frac{m}{k}$.

From (4.24), (4.25) we obtain

$x_{1}+x_{2}=-\frac{m-1}{k-1}$.

The relation is (4.31) is satisfied due to (4.32) and $m \neq k$.

Now we analyse the inequalities in (4.28). We introduce new parameter

$\varepsilon_{1}=\bar{\varepsilon}_{1} \operatorname{sign}(m-k)$.

Then relation (4.18) reads as follows

$X=\frac{k-1}{2(m+k-2)(m+2 k-3)}+\varepsilon_{1} \frac{\sqrt{D}}{2|A|}$,

$\varepsilon_{1}= \pm 1$.

Let us consider the case $\varepsilon_{1}=-1$. The second inequality in (4.28) $X<\frac{k-1}{(m-1)(m+2 k-3)}$ is obeyed since $2(m+k-2)>$ $m-1$. Now we consider the first inequality $X>0$. We get $0<\sqrt{D}<(m-1)|m-k|(k-1)$.

Using the definition of $D$ in (4.14) we obtain

$$
\begin{aligned}
0 & <(m-1)(m-k)(k-1)^{2}(F-8 \lambda f) \\
& <(m-1)^{2}|m-k|^{2}(k-1)^{2} .
\end{aligned}
$$

Relations (4.36) read as follows

$$
\begin{gathered}
F_{-}<8 \lambda f<F, \text { for } m>k, \\
F<8 \lambda f<F_{-}, \text {for } m<k,
\end{gathered}
$$

where

$F_{-} \equiv F-(m-1)(m-k)$. 
It may be verified that

$\frac{F_{-}}{8 f}=\frac{k}{8(k-1)}=\lambda_{\infty}=\lambda_{\infty}(k, k)$,

where $\lambda_{\infty}(k, l)$ is defined in (3.22). Using (4.23) and (4.40) we rewrite relations $(4.37),(4.38)$ as follows

$$
\begin{aligned}
& \lambda_{\infty}<\lambda<\lambda_{1}, \text { for } m>k, \\
& \lambda_{1}<\lambda<\lambda_{\infty}, \text { for } m<k .
\end{aligned}
$$

Now, we put $\varepsilon_{1}=1$. The inequality $X>0$ is satisfied in this case. We should treat the inequality $X<\frac{k-1}{(m-1)(m+2 k-3)}$. We obtain

$0<\sqrt{D}<|m-k|(m+2 k-3)(k-1)$,

or

$0<(m-1)(m-k)(F-8 \lambda f)<|m-k|^{2}(m+2 k-3)^{2}$.

Relations (4.44) read as follows

$$
\begin{aligned}
& F_{+}<8 \lambda f<F, \text { for } m>k, \\
& F<8 \lambda f<F_{+}, \text {for } m<k,
\end{aligned}
$$

where

$F_{+} \equiv F-(m-1)^{-1}(m-k)(m+2 k-3)^{2}$.

It may be verified that

$\frac{F_{+}}{8 f}=\lambda_{3}=\lambda_{3}(m, k, k)$,

where $\lambda_{3}(m, k, l)$ is defined in (3.11). Using (4.23) and (4.48) we rewrite relations $(4.45),(4.46)$ as follows

$$
\begin{aligned}
& \lambda_{3}<\lambda<\lambda_{1}, \text { for } m>k, \\
& \lambda_{1}<\lambda<\lambda_{3}, \text { for } m<k .
\end{aligned}
$$

We note that that

$\lambda_{1}<\lambda_{\infty}<\lambda_{3}$

for $m<k$ (it proved in the previous section), while

$\lambda_{3}<\lambda_{\infty}<\lambda_{1}$

for $k<m$. The inequalities in (4.52) follow from $F_{+}<$ $F_{-}<F$ for $k<m$.

Proposition 2 The solutions to equations (2.4), (2.5) for ansatz (2.7) with $1<m, 1<k_{1}=k_{2}=k, m \neq k$, obeying the inequalities $H \neq 0, H \neq h_{1}, H \neq h_{2}, h_{1} \neq h_{2}$, $S_{1}=m H+k h_{1}+k h_{2} \neq 0$ do exist if and only if $\alpha>0$,

$\lambda_{1}<\lambda=\alpha \Lambda<\lambda_{3}$

for $m<k$ and

$\lambda_{3}<\lambda=\alpha \Lambda<\lambda_{1}$, where $\lambda_{1}=\lambda_{1}(k, k), \lambda_{3}=\lambda_{3}(k, k)$ are defined in (3.9) and (3.11). In this case $H$ obeys the relation (4.6) with $X$ from (4.34), $x_{1}=h_{1} / H$ and $x_{2}=h_{2} / H$ are given by relations (4.24) and (4.25), $\lambda$ obeys (4.41), (4.42) for $\varepsilon_{1}=-1$ and (4.49), (4.50) for $\varepsilon_{1}=1$ with $\lambda_{\infty}=\frac{k}{8(k-1)}$.

The restrictions on $\lambda$ for our solution may be explained just graphically as it was done in the previous section for $k_{1} \neq k_{2}$. Indeed, for $k_{1}=k_{2}=k \neq m, H \neq 0$ we have the same relation (3.1) $\lambda=f\left(x_{1}\right)$, where now

$$
\frac{d f}{d x_{1}}=\frac{\bar{C}(m, k)\left(x_{1}-X_{1}\right)\left(x_{1}-X_{2}\right)\left(x_{1}-X_{3}\right)}{\left(-(k-1) \mathcal{P}\left(x_{1}, x_{2}\left(x_{1}\right)\right)\right)^{3}}
$$

with

$\bar{C}(m, k)=2(m-1)(k-1)^{3}(k-m)$.

Here $x_{2}\left(x_{1}\right)=-\frac{m-1}{k-1}-x_{1}$ and restrictions (2.17) reads as follows

$$
\begin{aligned}
& x_{1} \neq X_{1}=1, \quad x_{1} \neq X_{2}=-\frac{m+k-2}{k-1}, \\
& x_{1} \neq X_{3}=-\frac{m-1}{2 k-2},
\end{aligned}
$$

see (3.3)-(3.5). The fourth inequality in (2.17) is obeyed identically (it was checked above).

The points $X_{1}, X_{2}, X_{3}$ are points of extremum of the function $f\left(x_{1}\right)$. They are excluded from our consideration due to restrictions (4.57). The function $f\left(x_{1}\right)$ tends to $\lambda_{\infty}$ as $x_{1}$ tends to $\pm \infty$.

Using relations (4.55), (4.56) and $\mathcal{P}\left(x_{1}, x_{2}\left(x_{1}\right)\right)<0$ we get two cases.

For $1<m<k$ the function has two points of minimum at $X_{1}$ and $X_{2}$ with $\lambda_{1}=f\left(X_{1}\right)=f\left(X_{2}\right)=\lambda_{2}<\lambda_{\infty}$, and the point of maximum at $X_{3}$ with $\lambda_{3}=f\left(X_{3}\right)>\lambda_{\infty}$. See graphical representation of $f\left(x_{1}\right)$ for $m=4$ and $k=5$ at Fig. 4.

For $1<k<m$ the function has two points of maximum at $X_{1}$ and $X_{2}$ with $\lambda_{1}=f\left(X_{1}\right)=f\left(X_{2}\right)=\lambda_{2}>\lambda_{\infty}$, and one point of minimum at $X_{3}$ with $\lambda_{3}=f\left(X_{3}\right)<\lambda_{\infty}$. The graphical representation of $f\left(x_{1}\right)$ for $m=5$ and $k=4$ is depicted at Fig. 5.

We note that special solutions (e.g. stable ones) with $\left(m, k_{1}, k_{2}\right)=(3,4,4),(2,3,3),(4,3,3)$ were considered earlier in [35].

The case $H=0$. For $k_{1}=k_{2}=k>1$ and $H=0$ the solutions under consideration obeying restrictions (2.8) are absent. Indeed, using relations (2.9), (2.10) and (2.11), we get (see (3.57), (3.58) and (3.59)) $\alpha>0$,

$\alpha \Lambda=\frac{k}{8(k-1)}=\lambda_{\infty}$, 


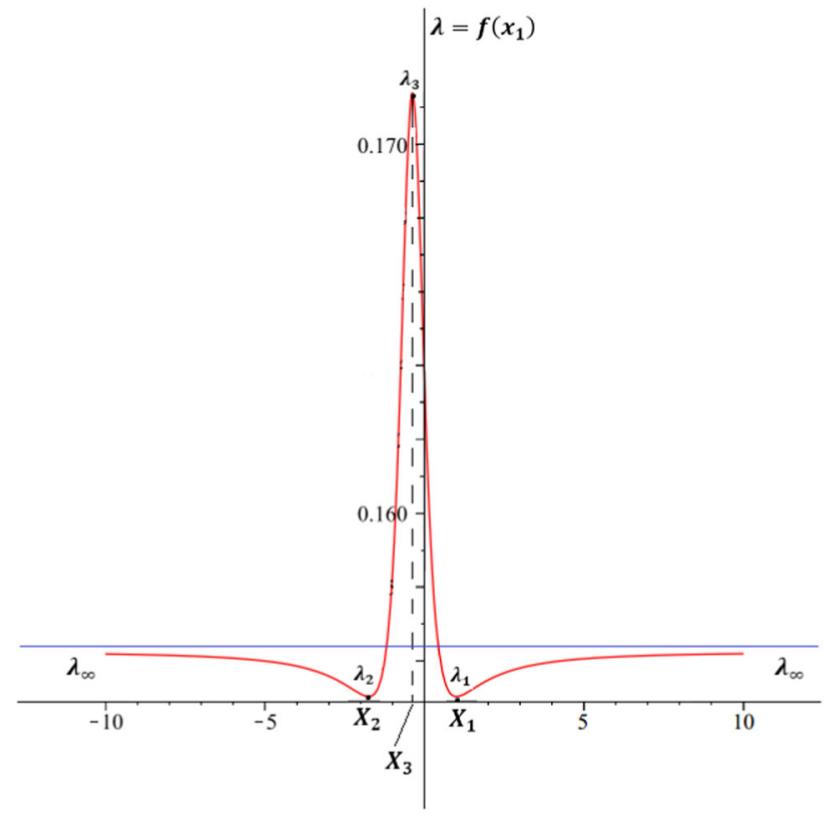

Fig. 4 The function $\lambda=f\left(x_{1}\right)$ for $m=4, k_{1}=k_{2}=5$

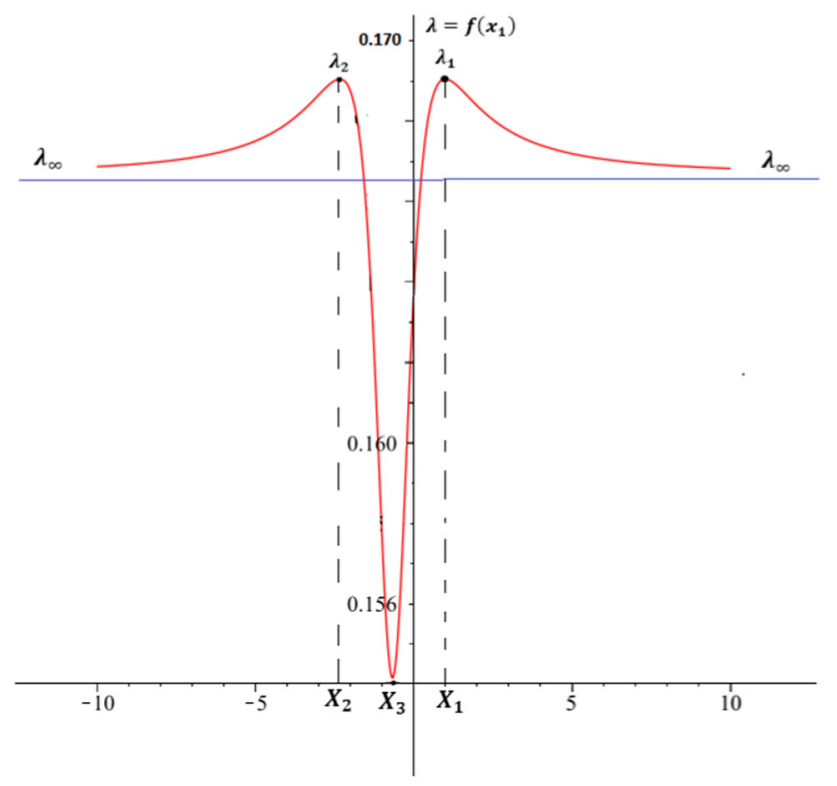

Fig. 5 The function $\lambda=f\left(x_{1}\right)$ for $m=5, k_{1}=k_{2}=4$

and

$h_{1}=-h_{2}= \pm \frac{1}{\sqrt{4 \alpha(k-1)}}$.

We obtain $S_{1}=k_{1} h_{1}+k_{2} h_{2}=0$, which is in contradiction with our restriction $S_{1} \neq 0$. Nevertheless, it may be verified that the Hubble-like parameters $H=0$ and $h_{1}, h_{2}$ from (4.59) obey the equations of motion (2.4), (2.5) for $\alpha>0$ and $\Lambda$ from (4.58). This means that we are led to a special solution, belonging to a subclass of solutions obeying $S_{1}=0$, which is out consideration in this paper.

\section{The analysis of stability}

Here we study the stability of the solutions under consideration by using the results of refs. [23,25,26].

We put the restriction

$\operatorname{det}\left(L_{i j}(v)\right) \neq 0$

on the matrix

$L=\left(L_{i j}(v)\right)=\left(2 G_{i j}-4 \alpha G_{i j k s} v^{k} v^{s}\right)$.

We remind that for general cosmological setup with the metric

$g=-d t \otimes d t+\sum_{i=1}^{n} e^{2 \beta^{i}(t)} d y^{i} \otimes d y^{i}$,

we have the set of equations [23]

$E=G_{i j} h^{i} h^{j}+2 \Lambda-\alpha G_{i j k l} h^{i} h^{j} h^{k} h^{l}=0$,

$Y_{i}=\frac{d L_{i}}{d t}+\left(\sum_{j=1}^{n} h^{j}\right) L_{i}-\frac{2}{3}\left(G_{s j} h^{s} h^{j}-4 \Lambda\right)=0$,

where $h^{i}=\dot{\beta}^{i}$,

$L_{i}=L_{i}(h)=2 G_{i j} h^{j}-\frac{4}{3} \alpha G_{i j k l} h^{j} h^{k} h^{l}$,

$i=1, \ldots, n$.

Due to results of Ref. [25] a fixed point solution $\left(h^{i}(t)\right)=$ $\left(v^{i}\right)(i=1, \ldots, n ; n>3)$ to eqs. (5.4), (5.5) obeying restrictions (5.1) is stable under perturbations

$h^{i}(t)=v^{i}+\delta h^{i}(t)$,

$i=1, \ldots, n$, as $t \rightarrow+\infty$, if (and only if)

$S_{1}(v)=\sum_{i=1}^{n} v^{i}>0$

and it is unstable if (and only if)

$S_{1}(v)=\sum_{i=1}^{n} v^{i}<0$.

In order to study the stability of solutions we should verify the relation (5.1) for the solutions under consideration. This verification was done (in fact) in Ref. [26]. The proof of Ref. [26] is based on first three relations in (2.8) and inequalities $k_{1}>1, k_{2}>1$ and $m>1$. We note the relation (2.14) was also used in this proof.

Thus, the any solution under consideration is stable when relation (5.8) is obeyed while it is unstable when relation (5.9) is satified. 
Let us consider the case $1<m<k_{1}<k_{2}$. For $H>0$ the relation $(5.8)$ reads as

$m+k_{1} x_{1}+k_{2} x_{2}=1+x_{1}+x_{2}>0$

or, equivalently,

$x_{1}>X_{4}=\frac{m-k_{2}}{k_{2}-k_{1}}$.

Here the equation (2.18) was used. For $H<0$ the stability condition (5.8) reads as

$m+k_{1} x_{1}+k_{2} x_{2}=1+x_{1}+x_{2}<0$,

or, equivalently, as

$x_{1}<X_{4}$.

The non-stability condition (5.9) reads as (5.13) for $H>0$ and as (5.11) for $H<0$.

Proposition 3 The solution to equations (2.4), (2.5) for ansatz (2.7) with $1<k_{1}<k_{2}$, obeying the inequalities $H \neq$ $0, H \neq h_{1}, H \neq h_{2}, h_{1} \neq h_{2}, S_{1}=m H+k_{1} h_{1}+k_{2} h_{2} \neq 0$ is stable if and only if $H\left(x_{1}-X_{4}\right)>0\left(X_{4}=\frac{m-k_{2}}{k_{2}-k_{1}}\right)$ and it is unstable if and only if $H\left(x_{1}-X_{4}\right)<0$.

Now we consider the case $H \neq 0,1<m, 1<k_{1}=$ $k_{2}=k, m \neq k$. The exact solutions obtained in this section obey first three relations in (2.8) (since $x_{1} \neq 1, x_{2} \neq 1$ and $x_{1} \neq x_{2}$ ) and hence the key restriction (5.1) is satisfied.

The stability condition (5.8) in this case reads as,

$$
\begin{aligned}
H\left(m+k_{1} x_{1}+k_{2} x_{2}\right) & =H\left(1+x_{1}+x_{2}\right) \\
& =H\left(1-\frac{m-1}{k-1}\right)>0,
\end{aligned}
$$

see (4.32), or, equivalently,

$H(k-m)>0$.

The non-stability condition (5.9) reads as

$H(k-m)<0$.

Thus, we are led to the proposition.

Proposition 4 The solution to equations (2.4), (2.5) for ansatz. (2.7) with $1<m, 1<k_{1}=k_{2}=k, m \neq k$, obeying the inequalities $H \neq 0, H \neq h_{1}, H \neq h_{2}$, $h_{1} \neq h_{2}, S_{1}=m H+k h_{1}+k h_{2} \neq 0$ is stable if and only if $H(k-m)>0$ and it is unstable if and only if $H(k-m)<0$.

For $H>0$ (or $\varepsilon_{0}=1$, see (4.6)) our special solutions are stable for $k>m$ and they are unstable for $k<m$. For $H<0$ (or $\varepsilon_{0}=-1$ ) the solutions are stable for $k<m$ and they are unstable for $k>m$.

The case $H=0$. Let us consider the solutions with $H=0$ and $h_{1}, h_{2}$ from (3.58), (3.59), which are valid for $k_{1} \neq k_{2}$, $\alpha>0$ and $\Lambda$ from (3.57). Here $k_{1}>1$ and $k_{2}>1$. We obtain

$$
\begin{aligned}
S_{1}= & k_{1} h_{1}+k_{2} h_{2}= \pm\left(k_{2}-k_{1}\right) \\
& \times\left(2 \alpha\left(k_{1}-1\right)\left(k_{2}-1\right)\left(k_{1}+k_{2}-2\right)\right)^{-1 / 2},
\end{aligned}
$$

where \pm is sign parameter in (3.58), (3.59). It follows from our analysis above that the solution with $\pm\left(k_{2}-k_{1}\right)>0$ is stable. This takes place when either $k_{2}>k_{1}$ and the sign " + " is chosen in (3.58) and (3.59), or if $k_{2}<k_{1}$ and the sign " - " is selected. For $\pm\left(k_{2}-k_{1}\right)<0$ the solution is unstable. Here the restriction $m>1$ (which is used for the proof of (5.1)) is also assumed.

\section{Solutions corresponding to zero variation of $G$}

Here we consider the special solutions to equations (2.9), (2.10), (2.11) with $H>0,3<m<k_{1}<k_{2}$ [26] (for $m=3$ see [36])

$h_{1}=\frac{m+2 k_{2}-3}{k_{2}-k_{1}} H, \quad h_{2}=\frac{m+2 k_{1}-3}{k_{1}-k_{2}} H$.

Here

$H=\left|k_{1}-k_{2}\right|(-2 \alpha P)^{-1 / 2}$,

$\alpha>0$,

$$
\begin{aligned}
P= & P\left(m, k_{1}, k_{2}\right)=-\left(m+k_{1}+k_{2}-3\right)\left(m\left(k_{1}+k_{2}-2\right)\right. \\
& \left.+k_{1}\left(2 k_{2}-5\right)+k_{2}\left(2 k_{1}-5\right)+6\right)<0,
\end{aligned}
$$

6 and

$\Lambda=\Lambda\left(m, k_{1}, k_{2}\right)$,

where

$$
\begin{aligned}
\Lambda\left(m, k_{1}, k_{2}\right)= & \frac{1}{8 \alpha P^{2}}\left(m+k_{1}+k_{2}-3\right) \\
& \times\left[\left(k_{1}+k_{2}\right)\left(k_{1}+k_{2}-2\right) m^{3}\right. \\
& +\left(k_{1}^{3}+k_{2}^{3}+11\left(k_{1}^{2} k_{2}+k_{1} k_{2}^{2}\right)-19\left(k_{1}^{2}+k_{2}^{2}\right)\right. \\
& \left.-22 k_{1} k_{2}+18\left(k_{1}+k_{2}\right)\right) m^{2} \\
& -\left(8 \left(\left(k_{1}^{3}+k_{2}^{3}\right)-63\left(k_{1}+k_{2}\right)^{2}-8 k_{1}^{2}\left(k_{1}-11\right) k_{2}\right.\right. \\
& \left.\left.-8 k_{2}^{2}\left(k_{2}-11\right) k_{1}\right)-32 k_{1}^{2} k_{2}^{2}+54\left(k_{1}+k_{2}\right)\right) m \\
& -\left(9\left(k_{1}^{3}+k_{2}^{3}\right)+45\left(k_{1}^{2}+k_{2}^{2}\right)-54\left(k_{1}+k_{2}\right)\right. \\
& +8\left(k_{1}^{2}+k_{2}^{2}\right) k_{1} k_{2} \\
& \left.\left.-16\left(k_{1}+k_{2}-10\right) k_{1}^{2} k_{2}^{2}-9\left(21 k_{1}+21 k_{2}-26\right) k_{1} k_{2}\right)\right] .
\end{aligned}
$$

These solutions describe accelerated exponential expansion of "our" $3 d$ subspace and constant internal space volume factor, or zero variation of the effective gravitational constant (in Jordan frame) obeying the most stringent limitation on $G$-dot obtained by the set of ephemerides [37], when the following splitting of the Hubble-like parameters is keeping in 
mind:

$v=(\underbrace{H, H, H}_{\text {"our" space }}, \underbrace{\overbrace{H, \ldots, H}^{m-3}, \overbrace{h_{1}, \ldots, h_{1}}^{k_{1}}, \overbrace{h_{2}, \ldots, h_{2}}^{k_{1}}}_{\text {internal space }})$.

It follows from Proposition 1 that $\Lambda\left(m, k_{1}, k_{2}\right)>0$. Moreover, in this case we have

$x_{1}=\frac{m+2 k_{2}-3}{k_{2}-k_{1}}>1$.

Due to graphical analysis from Sect. 3 we get from (6.7) the following bounds

$0<\lambda_{1}\left(m, k_{1}, k_{2}\right)<\Lambda\left(m, k_{1}, k_{2}\right) \alpha<\lambda_{\infty}\left(m, k_{1}, k_{2}\right)$

for all $3<m<k_{1}<k_{2}$.

Remark It may be also shown that the effective gravitational constant $G$ (in Jordan frame), calculated for our solutions, obeys the limitation on $G$-dot from Ref. [37], when $\Lambda$ belongs to some vicinity of $\Lambda\left(m, k_{1}, k_{2}\right)$, i.e. $\left|\Lambda-\Lambda\left(m, k_{1}, k_{2}\right)\right|<\delta$ for some (small enough) $\delta>0$.

\section{Hubble-like parameters vs. constants of the model}

The initial contants of the model are $\alpha_{1} \neq 0, \alpha_{2} \neq 0$ and $\Lambda$. The solutions for Hubble-like parameters $H \neq 0, h_{1}$ and $h_{2}$, which were analyzed above, depend upon $\alpha=\alpha_{2} / \alpha_{1}>0$ and $\lambda=\Lambda \alpha$. In this section we consider for simplicity the generic case $H \neq 0$. The parameter $\alpha$ has the dimension of $L^{2}$ ( $L$ is a length), while $\lambda$ is dimensionless one.

Here we discuss the existence of certain combinations of Hubble-like parameters, which either do not depend upon the parameters (or constants) of the model, i.e. $\alpha$ and $\lambda$, or depend only upon one of these constants. Such combinations (or functions) of $H \neq 0, h_{1}$ and $h_{2}$ do exist.

Indeed, it follows from (2.11) that the Hubble-like parameters for the solutions under consideration obey the following identity

$$
\begin{aligned}
\varphi_{1}\left(H, h_{1}, h_{2}\right) & \equiv(m-1) H+\left(k_{1}-1\right) h_{1}+\left(k_{2}-1\right) h_{2} \\
& =0,
\end{aligned}
$$

$m>1, k_{1}>1$ and $k_{2}>1$. This is the first basic relation (of this section). By using (2.20) and (2.23) we get the second basic relation

$$
\begin{aligned}
\varphi_{2}\left(H, h_{1}, h_{2}\right) & \equiv(m-1) H^{2}+\left(k_{1}-1\right) h_{1}^{2}+\left(k_{2}-1\right) h_{2}^{2} \\
& =\frac{1}{2 \alpha} .
\end{aligned}
$$

The third basic relation is just (3.1) which we rewrite here as

$\varphi_{3}\left(H, h_{1}, h_{2}\right) \equiv f\left(h_{1} / H\right)=\lambda$,

where $f\left(x_{1}\right)$ is the rational function defined in (3.1).

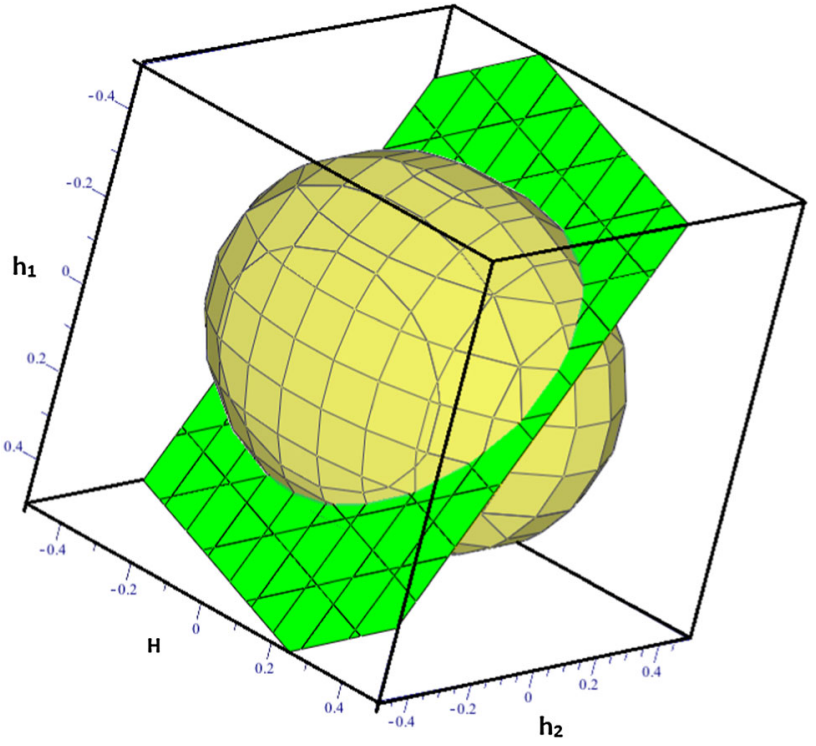

Fig. 6 The graphical representation (in Hubble-like variables $H, h_{1}, h_{2}$ ) of intersection of plane (see (7.1)) and ellipsoid (see (7.2)) for $m=3, k_{1}=4, k_{2}=5$ and $\alpha=1$

In the $3 d$ space of Hubble-like parameters $H, h_{1}, h_{2}$, relation (7.1) describes a plane while (7.2) corresponds to an ellipsoid. The intersection of this plane and ellipsoid gives us an ellipse $\mathcal{E}$. For $m=3, k_{1}=4$, $k_{2}=5$ and $\alpha=1$ this intersection is depicted at Fig. 6. For $H \neq 0$ and $m<k_{1}<k_{2}$ the solutions for $\left(H, h_{1}, h_{2}\right)$ are described by 1-dimensional manifold $\mathcal{E}_{\text {sol }}=\mathcal{E} \backslash\left\{N, S, Y_{1}, Y_{2}, Y_{3}, Y_{4},-Y_{1},-Y_{2},-Y_{3},-Y_{4}\right\}$, where points $N, S$ correspond to $H=0$, points $Y_{1}, Y_{2}, Y_{3}, Y_{4}$ correspond to $H>0$ and relations $h_{1} / H=X_{1}, h_{2} / H=$ $X_{2}, h_{3} / H_{3}=X_{3}, h_{4} / H_{4}=X_{4}$, respectively (see (3.3), (3.4), (3.5), (3.6)). Thus, the manifold $\mathcal{E}_{\text {sol }}$ is an 1dimensional manifold, which is obtained from the ellipse $\mathcal{E}$ by deleting 10 points. It is a disjoint union of ten arcs. Any of these arcs is parametrized by the pair $(\lambda, s)$, where $s$ is the number of the arc and $\lambda$ is local coordinate given by (7.3).

Analogous consideration may be done for the case $m \neq$ $k_{1}=k_{2}$ : in this case one should delete 8 points from $\mathcal{E}$ to obtain $\mathcal{E}_{\text {sol }}$.

It should be noted that (7.1) implies the following identity for scale factors $a_{i}(t)=\exp \left(h_{i} t+\beta_{i}\right), i=0,1,2,\left(h_{0}=H\right)$ $\left(a_{0}(t)\right)^{m-1}\left(a_{1}(t)\right)^{k_{1}-1}\left(a_{2}(t)\right)^{k_{2}-1}=$ const,

or

$$
\begin{aligned}
v(t) & =\left(a_{0}(t)\right)^{m}\left(a_{1}(t)\right)^{k_{1}}\left(a_{2}(t)\right)^{k_{2}} \\
& =\text { const } \times a_{0}(t) a_{1}(t) a_{2}(t) .
\end{aligned}
$$

Here $v(t)=\exp \left(\sum_{i=0}^{2}\left(h_{i} t+\beta_{i}\right)\right)$ is volume scale factor which is (exponentiallly) increasing in time for stable solutions (with $H+h_{1}+h_{2}>0$ ) and decreasing in time for unstable ones (with $H+h_{1}+h_{2}<0$ ). 


\section{Conclusions}

We have considered the $D$-dimensional Einstein-GaussBonnet (EGB) model with a $\Lambda$-term (or EGB $\Lambda$ model) and two (non-zero) constants $\alpha_{1}$ and $\alpha_{2}$. The metric was chosen to be diagonal "cosmological" one. Here we were dealing (mainly) with a class of solutions with exponential time dependence of three scale factors, governed by three noncoinciding Hubble-like parameters $H \neq 0, h_{1}$ and $h_{2}$, corresponding to factor spaces of dimensions $m>1, k_{1}>1$ and $k_{2}>1$, respectively, with the restriction imposed: $S_{1}=m H+k_{1} h_{1}+k_{2} h_{2} \neq 0$, and $D=1+m+k_{1}+k_{2}$.

We have studied the solutions in two cases: i) $m<k_{1}<k_{2}$ and ii) $1<k_{1}=k_{2}=k \neq m$. (The solutions under consideration with $k_{1}=k_{2}=m$ are absent.) We have shown that in both cases the solutions exist only if: $\alpha=\alpha_{2} / \alpha_{1}>0$, $\lambda=\alpha \Lambda>0$ and the dimensionless parameter of the model $\lambda$ obeys certain restrictions, e.g. upper and lower bounds depending upon $m, k_{1}$ and $k_{2}$ (see Proposition 1). In the case ii) we have found explicit exact solutions (see Proposition 2).

Our consideration used the so-called Chirkov-PavluchenkoToporensky splitting trick from Ref. [20] (see also [26]) which allowed us to reduce the problem under consideration to master equation $\lambda=f\left(x_{1}\right)$ (2.31), where $x_{1}=h_{1} / H$. This master equation is equivalent to polynomial equation (2.34) for $x_{1}$ which is of fourth order (in generic case) or less depending upon $\lambda$. Thus, the master equation may be solved in radicals for all $m>1, k_{1}>1$ and $k_{2}>1$. Our restrictions on $\lambda$ were obtained by analysing the equation $\lambda=f\left(x_{1}\right)$ with the use of the formulas for the derivative $d f / d x_{1}$, i.e. (3.7) and (4.55) in cases i) and ii), respectively. In the case i) $m<k_{1}<k_{2}$ the extremum points of the function $f\left(x_{1}\right)$ are just four non-coinciding points: $X_{1}, X_{2}, X_{3}, X_{4}$ (see (3.3), (3.4), (3.5), (3.6)) which are exactly four values of $x_{1}$ forbidden by restrictions $H \neq h_{1}, H \neq h_{2}, h_{1} \neq h_{2}$, $S_{1}=m H+k_{1} h_{1}+k_{2} h_{2} \neq 0$, respectively. In the case ii) $1<k_{1}=k_{2} \neq m$ we have three forbidden points: $X_{1}, X_{2}, X_{3}$.

The stability of the solutions (as $t \rightarrow+\infty$ ) in a class of cosmological solutions with diagonal metrics was analyzed for both cases ((i) and (ii)) and subclasses of stable and non-stable solutions were singled out. We have proved that in the case i) the solutions with $H>0$ are stable for $x_{1}=h_{1} / H>X_{4}=\frac{m-k_{2}}{k_{2}-k_{1}}$ and unstable for $x_{1}<X_{4}$ (see Proposition 3). It was proved that in the case ii) the solutions with $H>0$ are stable for $k>m$ and unstable for $k<m$ (see Proposition 4). The stability conditions for $H<0$ are equivalent to instability conditions for $H>0$ and vice versa. The solutions of first class i) contains a subclass of stable solutions describing an exponential expansion of 3-dimensional subspace with Hubble-like parameter $H>0$ and zero vari- ation of the effective gravitational constant $G$ (in the Jordan frame) [26] (see Sect. 6).

Some of the results obtained in this paper may be considered as non-trivial and unexpected ones. Indeed, let us compare the solutions governed by three different Hubblelike parameters $H>0, h_{1}, h_{2}$ with the solutions from Ref. [27] obtained for two non-coinciding Hubble-like parameters $H>0$ and $h$ corresponding to factor spaces of dimensions $m>2$ and $l>2$ with $m H+l h \neq 0$. Here we have found that our solutions take place only for $\alpha>0$ and $\Lambda>0$, while in the case of Ref. [27] we have two branches with (a) $\alpha>0$, $-\infty<\Lambda \alpha<\lambda_{+}(m, l)$ and (b) $\alpha<0, \Lambda|\alpha|>\lambda_{-}(m, l)$, where $\lambda_{ \pm}(m, l)>0$. The solutions from Ref. [27] with $\alpha>0$ exist for any $\Lambda \in(-\infty, 0]$, while in our case such solutions are absent. We note that the absence of solutions for $\Lambda=0$ may be considered as a special non-trivial result. For two different Hubble parameters such solutions (with $\Lambda=0$ and $\alpha>0$ ) were described in Ref. [38]. As it is proved here, in the case of three Hubble-like parameters (with the restrictions imposed above) the allowed gap for $\Lambda$ is bounded (at the top and the bottom).

Here we have also considered (for a completeness) the case $H=0$ and have found that the solutions exist only for $k_{1} \neq k_{2}, \alpha>0$ and fixed value of $\Lambda>0$ from (3.57). In this case we have two opposite in sign solutions for $\left(h_{1}, h_{2}\right)$ with one solution being stable and the second one - unstable.

For possible physical (e.g. cosmological) applications one may keep in mind a dimensional reduction of the model under consideration to $d=4$ which lead us to $4 d$ Horndeski type model with a set of scalar fields. In this case one will obtain $(1+3)$-dimensional inflationary (cosmological) solution with Hubble parameter $H>0$ and several scalar fields (coming from scale factors) with linear dependence upon the time variable (governed by $h_{1}$ and $h_{2}$ ). The effective cosmological term $\Lambda_{0}=3 H^{2}$ will have a nontrivial dependence upon the "bare" multidimensional cosmological constant $\Lambda$, the dimensions of factor spaces $m, k_{1}$ and $k_{2}$ and the parameter $\alpha$ (for any root of polynomial equation for $x_{1}$ ).

Acknowledgements The publication has been prepared with the support of the "RUDN University Program 5-100" (recipient V.D.I., mathematical model development). The reported study was funded by RFBR, project number 19-02-00346 (recipient K.K.E., simulation model development).

Data Availability Statement This manuscript has no associated data or the data will not be deposited. [Authors' comment: All data generated or analysed during this study are included in this published article.]

Open Access This article is licensed under a Creative Commons Attribution 4.0 International License, which permits use, sharing, adaptation, distribution and reproduction in any medium or format, as long as you give appropriate credit to the original author(s) and the source, provide a link to the Creative Commons licence, and indicate if changes were made. The images or other third party material in this article are included in the article's Creative Commons licence, unless indi- 
cated otherwise in a credit line to the material. If material is not included in the article's Creative Commons licence and your intended use is not permitted by statutory regulation or exceeds the permitted use, you will need to obtain permission directly from the copyright holder. To view a copy of this licence, visit http://creativecomm ons.org/licenses/by/4.0/.

Funded by SCOAP ${ }^{3}$.

\section{A Appendix}

Here we prove several technical lemmas.

\section{Lemma 1 Let}

$$
v(m, l, k)=(k+l) m^{2}+(m+l) k^{2}+(m+k) l^{2}-6 m l k,
$$

where $m, l, k$ are natural numbers. Then $v(m, l, k)=0$ only if $m=l=k$; in other cases $v(m, l, k)>0$.

Proof Since the $v(m, l, k)$ is symmetric in variables we put without loss of generality $m \geq l \geq k$. We have $m=k+p+q$, $l=k+p$, where $p \geq 0$ and $q \geq 0$. We get

$v=v(m, l, k)=\left(2 p^{2}+2 q p+2 q^{2}\right) k+2 p^{3}+3 q p^{2}+p q^{2}$.

For $p=q=0(m=k=l)$ we have $v=0$. For $p>0$, $q>0$ we have $v>0$. If $p=0(k=l)$ and $q>0(m>l)$ we get $v=2 q^{2} k>0$ for $k>1$. For $q=0(m=l)$ and $p>0(l>k)$ we find $v=2 p^{2} k+2 p^{3}>0$. The lemma is proved.

\section{Lemma 2 Let}

$$
\begin{aligned}
w(m, l, k)= & (k+l-2) m^{2}+(m+l-2) k^{2}+(m+k-2) l^{2} \\
& +2 k m+2 k m+2 l k-6 m l k,
\end{aligned}
$$

where $m, l, k$ are natural numbers non equal to 1 . Then $w(m, l, k)=0$ only if $m=l=k$. In other cases $w(m, l, k)>0$.

Proof Since the $w(m, l, k)$ is symmetric in variables we put without loss of generality $m \geq l \geq k$. We have $m=k+p+q$, $l=k+p$, where $p \geq 0$ and $q \geq 0$. We get

$$
\begin{aligned}
w= & w(m, l, k)=\left(2 p^{2}+2 q p+2 q^{2}\right)(k-1) \\
& +2 p^{3}+3 q p^{2}+q^{2} p .
\end{aligned}
$$

For $p=q=0(m=k=l)$ we have $w=0$. For $p>0$, $q>0$ we have $w>0$ (for all $k$ ). If $p=0(k=l)$ and $q>0$ $(m>l)$ we get $w=2 q^{2}(k-1)>0$ for $k>1$. For $q=0$ $(m=l)$ and $p>0(l>k)$ we find $w=2 p^{2}(k-1)+2 p^{3}>$ 0 . The lemma is proved.
Lemma 3 For all $1<m<k_{1}<k_{2}$

$$
\begin{aligned}
z_{1}= & \left(2 k_{1}-k_{2}-1\right) m-2 k_{1} k_{2}-4 k_{1} \\
& +5 k_{2}-k_{2}^{2}+2 k_{1}^{2}<0 .
\end{aligned}
$$

Proof Let us denote

$k_{1}=m+1+y_{1}, \quad k_{2}=k_{1}+1+y_{2}$.

Due to $m<k_{1}<k_{2}$ we get $y_{1} \geq 0$ and $y_{2} \geq 0$. The substitution of (A.5) into $z_{1}$ gives us

$$
\begin{aligned}
z_{1}= & -y_{2}^{2}+\left(-4 y_{1}-5 m-1\right) y_{2}-y_{1}^{2}+(-m-5) y_{1} \\
& -6 m \leq-6 m<0
\end{aligned}
$$

The lemma is proved.

Lemma 4 For all $1<m<k_{1}<k_{2}$

$$
\begin{aligned}
z_{3}= & -\left(k_{1}-k_{2}\right)^{2} m^{2}-\left(\left(k_{1}^{2}-6 k_{1}+6\right) k_{1}+\left(k_{2}^{2}-6 k_{2}+6\right) k_{2}\right. \\
& \left.+\left(k_{1}+k_{2}\right) k_{1} k_{2}-4\right) m-2\left(k_{1}+k_{2}\right) \\
& +\left(12-6 k_{1}-6 k_{2}+\left(k_{1}+k_{2}\right)^{2}\right) k_{1} k_{2}>0 .
\end{aligned}
$$

Proof Substituting (A.6) into $z_{3}$ we obtain

$$
\begin{aligned}
z_{3}= & \left(y_{1}+1\right) y_{2}^{3}+\left(5 y_{1}^{2}+(6 m+7) y_{1}+6 m+2\right) y_{2}^{2}+ \\
& \times\left(8 y_{1}^{3}+(18 m+16) y_{1}^{2}+\left(12 m^{2}+24 m+11\right) y_{1}\right. \\
& \left.+2 m^{3}+6 m^{2}+12 m+1\right) y_{2} \\
& +4 y_{1}^{4}+(12 m+12) y_{1}^{3}+\left(12 m^{2}+30 m+11\right) y_{1}^{2}+ \\
& \times\left(4 m^{3}+24 m^{2}+18 m+5\right) y_{1}+6 m^{3}+6 m^{2}+6 m \geq \\
& \times 6 m^{3}+6 m^{2}+6 m>0,
\end{aligned}
$$

since $y_{1} \geq 0$ and $y_{2} \geq 0$. The lemma is proved.

\section{References}

1. B. Zwiebach, Curvature squared terms and string theories. Phys. Lett. B 156, 315 (1985)

2. E.S. Fradkin, A.A. Tseytlin, Effective field theory from quantized strings. Phys. Lett. B 158, 316-322 (1985)

3. E.S. Fradkin, A.A. Tseytlin, Effective action approach to superstring theory. Phys. Lett. B 160, 69-76 (1985)

4. D. Gross, E. Witten, Superstrings modifications of Einstein's equations. Nucl. Phys. B 277, 1 (1986)

5. H. Ishihara, Cosmological solutions of the extended Einstein gravity with the Gauss-Bonnet term. Phys. Lett. B 179, 217 (1986)

6. N. Deruelle, On the approach to the cosmological singularity in quadratic theories of gravity: the Kasner regimes. Nucl. Phys. B 327, 253-266 (1989)

7. S. Nojiri, S.D. Odintsov, Introduction to modified gravity and gravitational alternative for Dark Energy. Int. J. Geom. Methods Mod. Phys. 4, 115-146 (2007). arXiv:hep-th/0601213

8. G. Cognola, E. Elizalde, S. Nojiri, S.D. Odintsov, S. Zerbini, Oneloop effective action for non-local modified Gauss-Bonnet gravity in de Sitter space. Eur. Phys. J. C 64(3), 483-494 (2009). arXiv: 0905.0543 
9. E. Elizalde, A.N. Makarenko, V.V. Obukhov, K.E. Osetrin, A.E. Filippov, Stationary vs. singular points in an accelerating FRW cosmology derived from six-dimensional Einstein-Gauss-Bonnet gravity. Phys. Lett. B 644, 1-6 (2007). arXiv:hep-th/0611213

10. K. Bamba, Z.-K. Guo, N. Ohta, Accelerating cosmologies in the Einstein-Gauss-Bonnet theory with dilaton. Prog. Theor. Phys. 118, 879-892 (2007). arXiv: 0707.4334

11. A. Toporensky, P. Tretyakov, Power-law anisotropic cosmological solution in 5+1 dimensional Gauss-Bonnet gravity. Grav. Cosmol. 13, 207-210 (2007). arXiv: 0705.1346

12. S.A. Pavluchenko, A.V. Toporensky, A note on differences between $(4+1)$ - and $(5+1)$-dimensional anisotropic cosmology in the presence of the Gauss-Bonnet term. Mod. Phys. Lett. A 24, 513521 (2009)

13. I.V. Kirnos, A.N. Makarenko, Accelerating cosmologies in Lovelock gravity with dilaton. Open Astron. J. 3, 37-48 (2010). arXiv: 0903.0083

14. S.A. Pavluchenko, On the general features of Bianchi-I cosmological models in Lovelock gravity. Phys. Rev. D 80, 107501 (2009). arXiv: 0906.0141

15. I.V. Kirnos, A.N. Makarenko, S.A. Pavluchenko, A.V. Toporensky, The nature of singularity in multidimensional anisotropic GaussBonnet cosmology with a perfect fluid. Gen. Relativ. Gravity 42, 2633-2641 (2010). arXiv: 0906.0140

16. V.D. Ivashchuk, On anisotropic Gauss-Bonnet cosmologies in ( + 1) dimensions, governed by an n-dimensional Finslerian 4-metric. Gravit. Cosmol. 16(2), 118-125 (2010). arXiv: 0909.5462

17. V.D. Ivashchuk, On cosmological-type solutions in multidimensional model with Gauss-Bonnet term. Int. J. Geom. Methods Mod. Phys. 7(5), 797-819 (2010). arXiv: 0910.3426

18. K.-I. Maeda, N. Ohta, Cosmic acceleration with a negative cosmological constant in higher dimensions. JHEP 1406, 095 (2014). arXiv: 1404.0561

19. D. Chirkov, S. Pavluchenko, A. Toporensky, Exact exponential solutions in Einstein-Gauss-Bonnet flat anisotropic cosmology. Mod. Phys. Lett. A 29, 1450093 (2014). (11 pages) arXiv: 1401.2962

20. D. Chirkov, S.A. Pavluchenko, A. Toporensky, Non-constant volume exponential solutions in higher-dimensional Lovelock cosmologies. Gen. Relativ. Gravity 47, 137 (2015). (33 pages) arXiv: 1501.04360

21. S.A. Pavluchenko, Stability analysis of exponential solutions in Lovelock cosmologies. Phys. Rev. D 92, 104017 (2015). arXiv: 1507.01871

22. S.A. Pavluchenko, Cosmological dynamics of spatially flat Einstein-Gauss-Bonnet models in various dimensions: lowdimensional $\Lambda$-term case. Phys. Rev. D 94, 084019 (2016). arXiv: 1607.07347

23. K.K. Ernazarov, V.D. Ivashchuk, A.A. Kobtsev, On exponential solutions in the Einstein-Gauss-Bonnet cosmology, stability and variation of G. Gravit. Cosmol. 22(3), 245-250 (2016)

24. F. Canfora, A. Giacomini, S.A. Pavluchenko, A. Toporensky, Friedmann dynamics recovered from compactified Einstein-GaussBonnet cosmology. arXiv:1605.00041
25. V.D. Ivashchuk, On stability of exponential cosmological solutions with non-static volume factor in the Einstein-Gauss-Bonnet model. Eur. Phys. J. C 76, 431 (2016). arXiv: 1607.01244v2

26. K.K. Ernazarov, V.D. Ivashchuk, Stable exponential cosmological solutions with zero variation of $\mathrm{G}$ and three different Hubble-like parameters in the Einstein-Gauss-Bonnet model with a $\Lambda$-term. Eur. Phys. J. C 77, 402 (2017). (7 pages) arXiv:1705.05456

27. V.D. Ivashchuk, A.A. Kobtsev, Stable exponential cosmological solutions with two factor spaces in the Einstein-Gauss-Bonnet model with a $\Lambda$-term. Gen. Relativ. Gravity 50, 119 (2018). arXiv: $1712.09703 \mathrm{v} 4$

28. S. Nojiri, S.D. Odintsov, V.K. Oikonomou, Modified gravity theories on a Nutshell: inflation, bounce and late-time evolution. Phys. Rept. 692, 1-104 (2017)

29. M. Benetti, S. Santos da Costa, S. Capozziello, J.S. Alcaniz, M. De Laurentis, Observational constraints on Gauss-Bonnet cosmology. Int. J. Mod. Phys. 27, 1850084 (2018)

30. S. Nojiri, S.D. Odintsov, V.K. Oikonomou, Unifying inflation with early and late-time dark energy in $F(R)$ gravity. arXiv:1912.13128

31. A.G. Riess et al., Observational evidence from supernovae for an accelerating universe and a cosmological constant. Astron. J. 116, 1009-1038 (1998)

32. S. Perlmutter et al., Measurements of omega and lambda from 42 high-redshift supernovae. Astrophys. J. 517, 565-586 (1999)

33. M. Kowalski, D. Rubin et al., Improved cosmological constraints from new, old and combined supernova datasets. Astrophys. J. 686(2), 749-778 (2008). arXiv: 0804.4142

34. D. Lovelock, The Einstein tensor and its generalizations. J. Math. Phys. 12, 498 (1971)

35. K.K. Ernazarov, V.D. Ivashchuk, Examples of stable exponential cosmological solutions with three factor spaces in EGB model with a $\Lambda$-term. Gravit. Cosmol. 25, 164-168 (2019)

36. K.K. Ernazarov, V.D. Ivashchuk, Stable exponential cosmological solutions with three different Hubble-like parameters in $(1+3+$ $\left.k_{1}+k_{2}\right)$-dimensional EGB model with a $\Lambda$-term. Symmetry $\mathbf{1 2}(2)$, 250 (2020). (24 pages)

37. E.V. Pitjeva, Updated IAA RAS planetary ephemerides-EPM2011 and their use in scientific research. Astron. Vestnik 47(5), 419-435 (2013). arXiv: 1308.6416

38. V.D. Ivashchuk, A.A. Kobtsev, Exponential cosmological solutions with two factor spaces in EGB model with $\Lambda=0$ revisited. Eur. Phys. J. C 79, 824 (2019) 